\title{
Novel Delay-Dependent Stability Criteria for Discrete-Time Neural Networks with Time-Varying Delay
}

\author{
Sreten Stojanovic $\mathbb{D}^{1},{ }^{1}$ Milan Stojanovic $\mathbb{D}^{2},{ }^{2,3}$ and Milos Stevanovic $\mathbb{D}^{1}$ \\ ${ }^{1}$ Department of Engineering Sciences and Applied Mathematics, University of Nis, Faculty of Technology, Bulevar oslobodjenja 124, \\ Leskovac 16000, Serbia \\ ${ }^{2}$ Department of System Control and Signal Processing, University of Belgrade, School of Electrical Engineering, Bulevar kralja \\ Aleksandra 73, Belgrade 11000, Serbia \\ ${ }^{3}$ Vlatacom Institute Ltd., Bulevar Milutina Milankovica 5, Belgrade 11000, Serbia
}

Correspondence should be addressed to Sreten Stojanovic; sstojanovic@tf.ni.ac.rs

Received 12 July 2018; Accepted 25 October 2018; Published 6 November 2018

Academic Editor: Xian-Ming Zhang

Copyright (C) 2018 Sreten Stojanovic et al. This is an open access article distributed under the Creative Commons Attribution License, which permits unrestricted use, distribution, and reproduction in any medium, provided the original work is properly cited.

\begin{abstract}
The delay-dependent stability problem is investigated for discrete-time neural networks with time-varying delays. A new augmented Lyapunov-Krasovskii functional (LKF) with single and double summation terms and several augmented vectors is proposed by decomposing the time-delay interval into two nonequidistant subintervals to derive less conservative stability conditions. Then, by using Wirtinger-based inequality, reciprocally, and extended reciprocally convex combination lemmas, tight estimations for sum terms in the forward difference of the LKF are given. Several zero equalities are introduced to further relax the existing results. Less conservative stability criteria are proposed in terms of linear matrix inequalities (LMIs). Finally, numerical examples are proposed to show the effectiveness and less conservativeness of the proposed method.
\end{abstract}

\section{Introduction}

During the past few decades, neural networks (NNs) have received great attention because of their wide applications in various fields such as image processing, signal processing, pattern recognition, associative memory, parallel computation, optimization, and error diagnosis $[1,2]$. One of the most important questions in theoretical analysis of NNs is dynamical behaviors of the NNs, such as their stability, periodic oscillatory, and chaos. Among them, analysis of the stability has received much attention and various stability conditions have been obtained in $[3,4]$.

It is well known that a time delay is inherent in various systems, including NNs, owing to the finite speed of signal transmission and conversion rate of the processors. Delays in a system may cause oscillation and divergence and further degrade the performance. It is of great importance to determine the admissible maximal delay bound such that the neural networks with a delay less than this upper bound remain stable. Hence, it is essential to investigate the stability of the neural networks with time delay [5-10].

Since most systems use a digital processor to acquire information from computers at discrete instants of time, it is essential to formulate discrete-time neural networks (DNNs) that are an analogue of continuous ones. Therefore, it is significant to study the dynamics of the DNNs and many results for the DNNs with time delay has received increasing attention; see [11-16] and references therein.

The stability criteria for the DNNs with time-varying delay can be classified into two categories: delay-independent stability criteria and delay-dependent ones [17, 18]. As is well known, delay-dependent stability criteria, which take advantage of the information on the size of time delays, are less conservative than delay-independent ones. The main aim of delay-dependent stability criteria is to get maximum delay bounds guaranteeing the addressed neural networks to be stable. In order to improve results regarding this problem, various methods have been applied to the delay-dependent 
category, such as augmented LKF method [13, 19-22], freeweighting matrix method $[18,23]$, summation inequality method [16, 24-27], delay-partitioning method [5, 28, 29], and reciprocally convex approach $[20,30,31]$.

It is known that the simple LKFs with the single and/or double sum terms lead to the criteria with high conservatism, while more augmented LKFs reduce conservatism but increase the number of decision variables. By constructing an augmented LKF with the sum terms of a state vector as well as the activation function, an improved stability condition for the asymptotic stability of DNNs was derived in [13] but with an enlarged number of decision variables. In recent years, the LKF including triple summation terms has also been applied to study the stability analysis of discrete-time DNNs for a further improvement of the results $[19,32]$. For the purpose of reducing conservatism, the delay-partitioning LKFs, in which the delay interval was separated into several subintervals, were introduced $[5,28,29]$.

Various free-weighting matrix-based stability criteria [18, 23] were established to improve the results obtained by the Jensen-based summation inequality. However, introducing the free-weighting matrices may result in increased number of decision variables.

After the computational complexity became one of the crucial aspects of a research in the area of the system stability, the direct bounding method based on summation inequalities once again becomes the most popular method $[17,19,32,33]$. Very recently, various types of the Wirtingerbased summation inequalities, tighter than the Jensen-based summation inequality, have been proposed for discrete-time linear time-delay systems $[25,34,35]$ and have also been used for the study of the discrete-time DNNs [36]. As one of useful methods to deal with the stability of delayed systems, the reciprocally convex approach was developed in [30] and has been extensively used to study the dynamical behaviours of time-delay systems since then (see [32, 37-39] and references therein).

Not long ago, an extended reciprocally convex combination lemma (ERCCL) has been developed $[26,31]$ to replace the popular reciprocally convex combination lemma (RCCL). It has a potential to reduce the conservatism of the RCCLbased criteria without introducing any extra decision variable due to its advantage of reduced estimation gap using the same decision variables.

Motivated by the aforementioned discussions, in this paper, the problem of delay-dependent stability for DNNs with interval time-varying delay is considered. The objective of the paper is to derive simple but efficient stability conditions for DNNs with interval time-varying delay. The major contributions of this paper are as follows. Delay-decomposition method is used with new augmented Lyapunov-Krasovskii functional, which contains several augmented vectors in single and double summation terms with a free parameter $\alpha$ that divides interval $\left[k-h_{2}, k-h_{1}\right]$ into two asymmetric subintervals $\left[k-h_{2}, k-\alpha-1\right]$ and $\left[k-\alpha, k-h_{1}\right]$. The value of parameter $\alpha$ is determined by solving linear matrix inequalities (LMIs) that are defined in the proposed stability criterion. In this way, a greater degree of freedom is enabled in estimating the stability of the DDN, which leads to a smaller conservative stability criterion. The convenient existing summations inequalities, as well as extended reciprocally convex combination lemma, have been used for calculation difference of KLF. In order to improve the stability criteria, the LKF is extended with an additional double summation term and zero equalities (ZEs) are introduced in calculation difference of LKF. As a result of applying the mentioned techniques, it is shown that the derived results are less conservative then the existing ones $[24,31,40,41]$.

Notations. Throughout the paper, $\mathbb{Z}^{+}$denotes the set of positive integers and $\mathfrak{R}^{n}$ denotes the $n$-dimensional Euclidean space and $\mathfrak{R}^{n \times m}$ the set of all $n \times m$ real matrices. For the positive integers $a$ and $b(b>a), \mathbb{Z}[a, b]$ denotes the set of all positive integers $z$ satisfying $a \leq z \leq b$. $I_{n}$ and $0_{n \times m}$ denote $n \times n$ identity matrix and $n \times m$ zero matrix, respectively. $X^{T}$ denotes the matrix transpose of $X$ and * represent the elements below the main diagonal of a symmetric matrix. $\operatorname{diag}\{a, b, \ldots, z\}$ denotes the block-diagonal matrix with elements $a, b, \ldots, z$ in the diagonal entries and $\operatorname{Sym}\{X\}=X+X^{T}$. For any symmetric matrix $X \in \Re^{n \times n}$ the notation $X>0(X \geq 0)$ means that $X$ is positive definite (positive semidefinite) matrix and $X<0(X \leq 0)$ means that $X$ is negative definite (negative semidefinite) matrix. For the matrices $A_{i} \in \Re^{n \times m}, i=1,2, \ldots, l, \operatorname{Col}\left\{A_{1}, A_{2}, \ldots, A_{l}\right\}$ denotes the column block matrix $\left[\begin{array}{llll}A_{1}^{T} & A_{2}^{T} & \cdots & A_{l}^{T}\end{array}\right]^{T}$. Matrices, if their dimensions are not explicitly stated, are assumed to be compatible for algebraic operations.

\section{Preliminaries}

Consider the discrete-time neural networks (DNNs) with interval time-varying delay of the form:

$$
\begin{aligned}
y(k+1)= & C y(k)+A g(y(k))+A_{d} g(y(k-h(k))) \\
& +J, \quad k \geq 0, \\
y(j)= & \varphi(j), \quad j \in\left\{-h_{2},-h_{2}+1, \ldots,-1,0\right\},
\end{aligned}
$$

where $k \in \mathbb{Z}^{+}, y(k) \in \mathfrak{R}^{n}$ is the neural state vector, $g(y(k))=\left[g_{1}\left(y_{1}(k)\right), g_{2}\left(y_{2}(k)\right), \ldots, g_{n}\left(y_{n}(k)\right)\right]^{T}$ denotes the neural activation functions, $\varphi(j)$ is the initial condition, $C=\operatorname{diag}\left\{c_{1}, c_{2}, \ldots, c_{n}\right\}, c_{i}>0$ represents the state feedback coefficient matrix, i.e., the rate with which the $i$-th neuron will reset its potential to the resting state in isolation when disconnected from the networks and external inputs, $A \in$ $\Re^{n \times n}$ and $A_{d} \in \Re^{n \times n}$ are the connection weight matrix and the delay connection weight matrix, respectively, and $J=\left[J_{1}, J_{2}, \ldots, J_{n}\right]^{T}$ is the external input vector. Integer $h(k)$ is time-varying delay that satisfies

$$
0<h_{1} \leq h(k) \leq h_{2},
$$

where $h_{1}$ and $h_{2}$ are known positive integers representing the lower and upper bounds of $h(k)$.

In this paper, the following assumption on the system (1) is made. 
Assumption 1 (see [18]). For any $a, b \in \mathfrak{R}$ and $a \neq b$, the continuous and bounded activation functions $g_{i}(\cdot)$ satisfy

$$
k_{i}^{-} \leq \frac{g_{i}(a)-g_{i}(b)}{a-b} \leq k_{i}^{+}, \quad i=1,2, \ldots, n,
$$

where $k_{i}^{-}$and $k_{i}^{+}$are known constants.

Since the activation function $g_{i}(\cdot)$ satisfies (3), the system (1) has an equilibrium point. Let us denote the equilibrium point by $y^{*}=\left[y_{1}^{*}, \cdots, y_{n}^{*}\right]^{T}$, and then $y^{*}=C y^{*}+A g\left(y^{*}\right)+$ $A_{d} g\left(y^{*}\right)+J$. In order to simplify our consideration, we shift the equilibrium point of the system (1) to the origin. Let $x_{i}(k)=y_{i}(k)-y_{i}^{*}$ and $f_{i}\left(x_{i}(k)\right)=g_{i}\left(x_{i}(k)+y_{i}^{*}\right)-g_{i}\left(y_{i}^{*}\right)$. Then, system (1) can be transformed to the following form:

$$
\begin{array}{r}
x(k+1)=C x(k)+A f(x(k))+A_{d} f(x(k-h(k))), \\
k \geq 0, \\
x(j)=\phi(j), \quad j \in\left\{-h_{2},-h_{2}+1, \ldots,-1,0\right\},
\end{array}
$$

where $x(k) \in \mathfrak{R}^{n}$ is the state vector of the transformed system and $\phi(j)$ is the initial condition.

After applying Assumption 1, one can check that the function $f(\cdot)$ with $f(0)=0$ satisfies

$$
k_{i}^{-} \leq \frac{f_{i}(a)-f_{i}(b)}{a-b} \leq k_{i}^{+}, \quad i=1,2, \ldots, n
$$

for all $a \neq b$. If $=0$, we have

$$
k_{i}^{-} \leq \frac{f_{i}(a)}{a} \leq k_{i}^{+}, \quad i=1,2, \ldots, n,
$$

for all $a \neq 0$.

The following lemmas will be used in the sequel to establish the main results.

Lemma 2 (Jensen's inequality [42]). For any positive definite symmetric matrix $R \in \mathfrak{R}^{n \times n}$ and two positive integers $a$ and $b>a$, the sum term $\sum_{j=a}^{b-1} x^{T}(j) R x(j)$ is estimated as

$$
(b-a) \sum_{j=a}^{b-1} x^{T}(j) R x(j) \geq \sum_{j=a}^{b-1} x^{T}(j) R \sum_{j=a}^{b-1} x(j) .
$$

Lemma 3 (Wirtinger-based inequality [25]). For a given positive definite symmetric matrix $R$, two positive integers a and $b>a$, and any sequence of discrete-time variable $x$ : $\mathbb{Z}[a, b] \longrightarrow \mathfrak{R}^{n}$, the following inequality holds:

$$
\begin{aligned}
(b-a) \sum_{i=a}^{b-1} \eta^{T}(i) R \eta(i) & \geq\left[\begin{array}{l}
\theta_{1} \\
\theta_{2}
\end{array}\right]^{T}\left[\begin{array}{cc}
R & 0 \\
* & 3 \rho(a, b) R
\end{array}\right]\left[\begin{array}{l}
\theta_{1} \\
\theta_{2}
\end{array}\right] \\
& \geq\left[\begin{array}{c}
\theta_{1} \\
\theta_{2}
\end{array}\right]^{T}\left[\begin{array}{cc}
R & 0 \\
* & 3 R
\end{array}\right]\left[\begin{array}{l}
\theta_{1} \\
\theta_{2}
\end{array}\right],
\end{aligned}
$$

where

$$
\begin{aligned}
\eta(k) & =x(k+1)-x(k), \\
\theta_{1} & =x(b)-x(a), \\
\theta_{2} & =x(b)+x(a)-\frac{2}{b-a+1} \sum_{i=a}^{b} x(i), \\
\rho(a, b) & = \begin{cases}\frac{(b-a+1)}{(b-a-1)}, & b-a \neq 1 \\
1, & b-a=1 .\end{cases}
\end{aligned}
$$

Remark 4. In [27], stability criteria for discrete-time delay systems are considered by using a new summation inequality with three slack matrix variables. It has been shown that inequality (8) is obtained as a special case of the new summation inequality with convenient selected values of the slack matrix variables. Therefore, by using the mentioned summation inequality, the conservatism can be reduced, but the introduction of three slack matrix variables can increase the computation complexity of the obtained stability criterion.

The reciprocally convex combination lemma (RCCL) plays an important role in the estimation of the forward difference of LKF.

Lemma 5 (reciprocally convex combination lemma [30, 31, 43]). For a real scalar $\delta \in(0,1)$, a symmetric matrix $R>0$, and any matrix $S$ satisfying

$$
\left[\begin{array}{ll}
R & S \\
* & R
\end{array}\right] \geq 0
$$

the following inequality holds:

$$
\left[\begin{array}{cc}
\frac{1}{\delta} R & 0 \\
* & \frac{1}{1-\delta} R
\end{array}\right] \geq\left[\begin{array}{ll}
R & S \\
* & R
\end{array}\right]
$$

An extended reciprocally convex combination lemma (ERCCL), which estimates the sum terms in the forward difference of the LKF tightly than RCCL, is presented as follows.

Lemma 6 (extended reciprocally convex combination lemma $[31,40])$. For a real scalar $\delta \in(0,1)$, a symmetric matrix $R>$ 0 , and any matrix $S$, the following inequality holds:

$$
\left[\begin{array}{cc}
\frac{1}{\delta} R & 0 \\
* & \frac{1}{1-\delta} R
\end{array}\right] \geq\left[\begin{array}{cc}
R+(1-\delta) T_{1} & S \\
* & R+\delta T_{2}
\end{array}\right],
$$

where $T_{1}=R-S R^{-1} S^{T}$ and $T_{2}=R-S^{T} R^{-1} S$.

Remark 7. Unlike RCCL, the condition (11) is not used in ERCCL. Thus, the matrix $S$ in ERCCL can be chosen more freely then the one in RCCL such that the feasibility criterion 
based on (13) is better than one based on (11). Further, if (11) holds then $T_{i} \geq 0, i=1,2$, and

$$
\left[\begin{array}{cc}
R+(1-\delta) T_{1} & S \\
* & R+\delta T_{2}
\end{array}\right] \geq\left[\begin{array}{cc}
R & S \\
* & R
\end{array}\right]
$$

Thus, the terms $(1-\delta) T$ and $\delta T_{2}$, which appear in (13), reduce estimation gap between two sides of (13) and inequality (13) in ERCCL is tighter then inequality (12) in RCCL. Note that the RCCL and ERCCL require the same number of decision variables ( $R$ and $S)$.

Remark 8. In [20], the specific reciprocally convex inequality is proposed. For comparison, we rewrite the result of Theorem 1 in [20] in a block form as

$$
\begin{aligned}
& {\left[\begin{array}{cc}
\frac{1}{\delta} R_{1} & 0 \\
* & \frac{1}{1-\delta} R_{2}
\end{array}\right]} \\
& \geq\left[\begin{array}{cc}
R_{1}+(1-\delta) T_{1} & \delta S_{1}+(1-\delta) S_{2} \\
* & R_{2}+\delta T_{2}
\end{array}\right],
\end{aligned}
$$

where $T_{1}=R_{1}-Y_{1} R_{2}^{-1} Y_{1}^{T}$ and $T_{2}=R_{2}-Y_{2}^{T} R_{1}^{-1} Y_{2}$. If taking $R_{1}=R_{2}=R$ and $S_{1}=S_{2}=S$, inequality (15) immediately reduces to (13), which means that ERCCL (Lemma 6) is a special case of Theorem 1 in [20]. During the calculation of the difference of LKF in (31), the inequality (13) is used instead of (15), because the same matrix $R=\widetilde{Z}_{2}$ appears twice on the block diagonal in (31), so that the condition $R_{1}=R_{2}=R$ is automatically satisfied.

Further, by applying inequality (13) to (32), only one matrix $S$ was used instead of two $\left(S_{1}\right.$ and $\left.S_{2}\right)$, as in the case of application of inequality (15). Thereby, a computation complexity of the proposed stability criterion (13) is decreased, but there is a possibility of increasing conservatism (13) in regard to (15).

Lemma 9 (see [40]). For a positive definite symmetric matrix $R$ and matrices $\Upsilon$ and $\Xi$, the following statements are equivalent:

(i) $\Xi-\Upsilon^{T} R \Upsilon<0$

(ii) There exists a matrix $\Psi$ with appropriate dimension such that

$$
\left[\begin{array}{cc}
\Xi+\operatorname{Sym}\left\{\Upsilon^{T} \Psi\right\} & \Psi^{T} \\
* & -R
\end{array}\right]<0 .
$$

\section{Main Results}

In this section, we will establish a new criterion on asymptotical stability for the discrete-time neural networks with interval time-varying delay (1).

We introduce the following notations for later use:

$$
\begin{aligned}
& \xi(k) \\
& =\left[\begin{array}{lllllllllll}
x^{T}(k) & x^{T}\left(k-h_{1}\right) & x^{T}(k-h(k)) & x^{T}\left(k-h_{2}\right) & x^{T}(k-\alpha) & v_{1}^{T}(k) & v_{2}^{T}(k) & v_{3}^{T}(k) & v_{4}^{T}(k) & f^{T}(x(k)) & f^{T}(x(k-h(k)))
\end{array}\right]^{T}, \\
& \eta(k)=x(k+1)-x(k), \\
& \zeta_{1}(k)=\left[x^{T}(k) \sum_{j=k-h_{1}}^{k-1} x^{T}(j) \sum_{j=k-h_{2}}^{k-h_{1}-1} x^{T}(j) \sum_{j=k-\alpha}^{k-h_{1}-1} x^{T}(j)\right]^{T}, \\
& \zeta_{2}(k)=\left[\begin{array}{ll}
x^{T}(k) & f^{T}(x(k))
\end{array}\right]^{T} \\
& \xi_{3}(k)=\left[\begin{array}{lll}
x^{T}(k) & \eta^{T}(k)
\end{array}\right]^{T}, \\
& v_{1}(k)=\sum_{j=k-h_{1}}^{k} \frac{x(j)}{h_{1}+1}, \\
& v_{2}(k)=\sum_{j=k-h(k)}^{k-h_{1}} \frac{x(j)}{h(k)-h_{1}+1}, \\
& v_{3}(k)=\sum_{j=k-h_{2}}^{k-h(k)} \frac{x(j)}{h_{2}-h(k)+1}, \\
& v_{4}(k)=\sum_{j=k-\alpha}^{k-h_{1}} \frac{x(j)}{\alpha-h_{1}+1}, \\
& \omega_{1}=\left(e_{1}-e_{2}\right) \xi(k) \text {, } \\
& \omega_{2}=\left(e_{1}+e_{2}-2 e_{6}\right) \xi(k) \text {, }
\end{aligned}
$$




$$
\begin{aligned}
& \omega_{3}=\left(e_{2}-e_{5}\right) \xi(k) \text {, } \\
& \omega_{4}=\left(e_{2}+e_{5}-2 e_{9}\right) \xi(k) \text {, } \\
& e_{i}=\left[0_{n \times(i-1) n}, I_{n}, 0_{n \times(11-i) n}\right], \quad i=1,2, \ldots, 11, \\
& e_{s}=C e_{1}+A e_{10}+A_{d} e_{11} \text {, } \\
& \rho(a, b)= \begin{cases}\frac{b-a+1}{b-a-1}, & b-a>1 \\
1, & b-a=1,\end{cases} \\
& \delta_{h}=\frac{h(k)-h_{1}}{h_{12}}, \quad h_{12}=h_{2}-h_{1} \text {, } \\
& \Sigma(h(k), \alpha)=\Sigma_{11}+\Sigma_{12}(h(k), \alpha)+\Sigma_{2}+\Sigma_{3}+\Sigma_{41}-\Sigma_{42}-\Sigma_{43}(h(k))+\Sigma_{51}(\alpha)-\Sigma_{52}(\alpha)-\Sigma_{S}, \\
& \Sigma_{11}=\Pi_{1}^{T} P \Pi_{1}-\Pi_{2}^{T} P \Pi_{2} \text {, } \\
& \Pi_{1}=\left[\begin{array}{c}
e_{s} \\
\left(h_{1}+1\right) e_{6}-e_{2} \\
-e_{3}-e_{4} \\
-e_{5}
\end{array}\right] \text {, } \\
& \Pi_{2}=\left[\begin{array}{c}
e_{1} \\
\left(h_{1}+1\right) e_{6}-e_{1} \\
-e_{2}-e_{3} \\
-e_{2}
\end{array}\right] \\
& \Sigma_{12}(h(k), \alpha)=\operatorname{Sym}\left\{\left(\Pi_{1}-\Pi_{2}\right)^{T} P \Pi(h(k), \alpha)\right\}, \\
& \Pi(h(k), \alpha)=\left[\begin{array}{c}
0 \\
0 \\
\left(h(k)-h_{1}+1\right) e_{7}+\left(h_{2}-h(k)+1\right) e_{8} \\
\left(\alpha-h_{1}+1\right) e_{9}
\end{array}\right], \\
& \Sigma_{2}=e_{1}^{T} Q_{1} e_{1}+e_{2}^{T}\left(Q_{3}-Q_{1}\right) e_{2}+e_{5}^{T}\left(Q_{2}-Q_{3}\right) e_{5}-e_{4}^{T} Q_{2} e_{4}, \\
& \Sigma_{3}=\left[\begin{array}{c}
e_{1} \\
e_{10}
\end{array}\right]^{T}\left(h_{12}+1\right) R\left[\begin{array}{c}
e_{1} \\
e_{10}
\end{array}\right]-\left[\begin{array}{c}
e_{3} \\
e_{11}
\end{array}\right]^{T} R\left[\begin{array}{l}
e_{3} \\
e_{11}
\end{array}\right], \\
& \Sigma_{4}(h(k))=\Sigma_{41}-\Sigma_{42}-\Sigma_{43}(h(k)), \\
& \Sigma_{41}=\left(e_{s}-e_{1}\right)^{T}\left(h_{1}^{2} Z_{1}+h_{12}^{2} Z_{2}\right)\left(e_{s}-e_{1}\right), \\
& \Sigma_{42}=E_{1}^{T}\left[\begin{array}{cc}
Z_{1} & 0 \\
* & 3 \rho\left(0, h_{1}\right) Z_{1}
\end{array}\right] E_{1}, \quad E_{1}=\left[\begin{array}{c}
e_{1}-e_{2} \\
e_{1}+e_{2}-2 e_{6}
\end{array}\right], \\
& \Sigma_{43}(h(k))=\left[\begin{array}{l}
E_{2} \\
E_{3}
\end{array}\right]^{T}\left(\left[\begin{array}{cc}
\widetilde{Z}_{2} & S \\
* & \widetilde{Z}_{2}
\end{array}\right]+\left[\begin{array}{cc}
\left(1-\delta_{h}\right) T_{1} & 0 \\
* & \delta_{h} T_{2}
\end{array}\right]\right)\left[\begin{array}{l}
E_{2} \\
E_{3}
\end{array}\right], \\
& T_{1}=\widetilde{Z}_{2}-S \widetilde{Z}_{2}^{-1} S^{T} \\
& T_{2}=\widetilde{Z}_{2}-S^{T} \widetilde{Z}_{2}^{-1} S \\
& \widetilde{\Sigma}_{43}\left(h_{1}\right)=\left[\begin{array}{c}
E_{2} \\
E_{3}
\end{array}\right]^{T}\left[\begin{array}{cc}
\widetilde{Z}_{2} & S \\
* & \widetilde{Z}_{2}
\end{array}\right]\left[\begin{array}{l}
E_{2} \\
E_{3}
\end{array}\right]+E_{2}^{T} \widetilde{Z}_{2} E_{2},
\end{aligned}
$$




$$
\begin{aligned}
& \widetilde{\Sigma}_{43}\left(h_{2}\right)=\left[\begin{array}{l}
E_{2} \\
E_{3}
\end{array}\right]^{T}\left[\begin{array}{cc}
\widetilde{Z}_{2} & S \\
* & \widetilde{Z}_{2}
\end{array}\right]\left[\begin{array}{l}
E_{2} \\
E_{3}
\end{array}\right]+E_{3}^{T} \widetilde{Z}_{2} E_{3}, \\
& \widetilde{Z}_{2}=\left[\begin{array}{cc}
Z_{2} & 0 \\
* & 3 Z_{2}
\end{array}\right], \\
& E_{2}=\left[\begin{array}{c}
e_{2}-e_{3} \\
e_{2}+e_{3}-2 e_{7}
\end{array}\right], \\
& E_{3}=\left[\begin{array}{c}
e_{3}-e_{4} \\
e_{3}+e_{4}-2 e_{8}
\end{array}\right], \\
& E_{4}=\left[\begin{array}{c}
e_{2}-e_{5} \\
e_{2}+e_{5}-2 e_{9}
\end{array}\right], \\
& E_{5}(h(k))=\left[\begin{array}{c}
\left(h(k)-h_{1}+1\right) e_{7}-e_{2} \\
e_{2}-e_{3}
\end{array}\right], \\
& E_{6}(h(k))=\left[\begin{array}{cc}
\left(h_{2}-h(k)+1\right) e_{8}-e_{3} \\
\Sigma_{52}(\alpha)=E_{4}^{T}\left[\begin{array}{cc}
Z_{3} & 0 \\
* & 3 \rho\left(h_{1}, \alpha\right) Z_{3}
\end{array}\right], \\
E_{56}(h(k))=\left[\begin{array}{cc}
E_{5}(h(k)) \\
E_{6}(h(k))
\end{array}\right], \\
\Sigma_{51}(\alpha)=\left(e_{s}-e_{1}\right)^{T}\left(\alpha-h_{1}\right)^{2} Z_{3}\left(e_{s}-e_{1}\right), \\
0
\end{array}\right],
\end{aligned}
$$

$\Sigma_{S}$$$
=\operatorname{Sym}\left\{\left(e_{10}-K_{1} e_{1}\right)^{T} M_{1}\left(e_{10}-K_{2} e_{1}\right)\right\}+\operatorname{Sym}\left\{\left(e_{11}-K_{1} e_{3}\right)^{T} M_{2}\left(e_{11}-K_{2} e_{3}\right)\right\}+\operatorname{Sym}\left\{\begin{array}{c}
\left(e_{10}-e_{11}-K_{1}\left(e_{1}-e_{3}\right)\right)^{T} M_{3} \times \\
\times\left(e_{10}-e_{11}-K_{2}\left(e_{1}-e_{3}\right)\right)
\end{array}\right\},
$$$$
\Sigma_{6}=\Sigma_{61}-\Sigma_{62}(h(k)),
$$$$
\Sigma_{61}=h_{12}^{2}\left[\begin{array}{c}
e_{1} \\
e_{s}-e_{1}
\end{array}\right]^{T} G\left[\begin{array}{c}
e_{1} \\
e_{s}-e_{1}
\end{array}\right]+h_{12} e_{2}^{T} H_{1} e_{2}-h_{12} e_{4}^{T} H_{2} e_{4}-h_{12} e_{3}^{T}\left(H_{1}-H_{2}\right) e_{3},
$$$$
\Sigma_{62}(h(k))=E_{56}^{T}(h(k)) G_{12} E_{56}(h(k)) \text {, }
$$$$
G_{i}=G+\left[\begin{array}{cc}
0 & H_{i} \\
* & H_{i}
\end{array}\right], \quad i=1,2,
$$$$
G_{12}=\left[\begin{array}{cc}
G_{1} & X \\
* & G_{2}
\end{array}\right]
$$$$
K_{1}=\operatorname{diag}\left\{k_{1}^{-}, k_{2}^{-}, \ldots, k_{n}^{-}\right\} \text {, }
$$$$
K_{2}=\operatorname{diag}\left\{k_{1}^{+}, k_{2}^{+}, \ldots, k_{n}^{+}\right\} \text {. }
$$

Theorem 10. For given positive integers $h_{1}$ and $h_{2}$, system (4) with interval time-varying delay satisfying condition (2) is asymptotically stable, if there exist positive-definite matrices $P \in \mathfrak{R}^{4 n \times 4 n}, Q_{i} \in \mathfrak{R}^{n \times n}, i=1,2,3, R \in \mathfrak{R}^{2 n \times 2 n}, Z_{i} \in \mathfrak{R}^{n \times n}$, $i=1,2,3$, positive definite diagonal matrices $M_{i} \in \mathfrak{R}^{n \times n}$, $i=1,2,3$, and any matrix $S \in \Re^{2 n \times 2 n}$ such that the following LMIs hold:

$$
\left[\begin{array}{cc}
\Phi\left(h_{1}, \alpha\right) & E_{2}^{T} S \\
* & -\widetilde{Z}_{2}
\end{array}\right]<0,
$$




$$
\left[\begin{array}{cc}
\Phi\left(h_{2}, \alpha\right) & E_{3}^{T} S^{T} \\
* & -\widetilde{Z}_{2}
\end{array}\right]<0,
$$

where

$$
\begin{gathered}
\Phi\left(h_{i}, \alpha\right)=\Sigma_{11}+\Sigma_{12}\left(h_{i}, \alpha\right)+\Sigma_{2}+\Sigma_{3}+\Sigma_{41}-\Sigma_{42} \\
-\widetilde{\Sigma}_{43}\left(h_{i}\right)+\Sigma_{51}(\alpha)-\Sigma_{52}(\alpha)-\Sigma_{s},
\end{gathered}
$$

$$
i=1,2 \text {. }
$$

Proof. Construct a LKF for the DNN (4) as follows:

$$
V(k)=\sum_{i=1}^{5} V_{i}(k),
$$

where

$$
\begin{aligned}
V_{1}(k)= & \zeta_{1}^{T}(k) P \zeta_{1}(k), \\
V_{2}(k)= & \sum_{j=k-h_{1}}^{k-1} x^{T}(j) Q_{1} x(j)+\sum_{j=k-h_{2}}^{k-\alpha-1} x^{T}(j) Q_{2} x(j) \\
& +\sum_{j=k-\alpha}^{k-h_{1}-1} x^{T}(j) Q_{3} x(j), \\
V_{3}(k)= & \sum_{j=k-h(k)}^{k-1} \zeta_{2}^{T}(j) R \zeta_{2}(j) \\
& +\sum_{i=-h_{2}+1}^{-h_{1}} \sum_{j=k+i}^{k-1} \zeta_{2}^{T}(j) R \zeta_{2}(j) \\
V_{4}(k)= & h_{1} \sum_{i=-h_{1}}^{-1} \sum_{j=k+i}^{k-1} \eta^{T}(j) Z_{1} \eta(j) \\
& +h_{12} \sum_{i=-h_{2}}^{-h_{1}-1} \sum_{j=k+i}^{k-1} \eta^{T}(j) Z_{2} \eta(j) \\
V_{5}(k)= & \left(\alpha-h_{1}\right) \sum_{i=-\alpha}^{-h_{1}-1} \sum_{j=k+i}^{k-1} \eta^{T}(j) Z_{3} \eta(j) .
\end{aligned}
$$

The forward difference of $\operatorname{LKF}(21), \Delta V(k)=V(k+1)-V(k)$, along the trajectories of the DNN (4) gives

$$
\Delta V_{1}(k)=\zeta_{1}^{T}(k+1) P \zeta_{1}(k+1)-\zeta_{1}^{T}(k) P \zeta_{1}(k) .
$$

Since

$$
\begin{aligned}
& \zeta_{1}(k) \\
& =\operatorname{Col}\left\{\begin{array}{c}
e_{1},\left(h_{1}+1\right) e_{6}-e_{1}, \\
\left(h(k)-h_{1}+1\right) e_{7}+\left(h_{2}-h(k)+1\right) e_{8} \\
-e_{2}-e_{3},\left(\alpha-h_{1}+1\right) e_{9}-e_{2}
\end{array}\right\} \xi(k) \\
& =\left(\Pi_{2}+\Pi(h(k), \alpha)\right) \xi(k),
\end{aligned}
$$

$$
\begin{aligned}
& \zeta_{1}(k+1) \\
& =C o l\left\{\begin{array}{c}
e_{s},\left(h_{1}+1\right) e_{6}-e_{2}, \\
\left(h(k)-h_{1}+1\right) e_{7} \\
\left(h_{2}-h(k)+1\right) e_{8}-e_{3}-e_{4}, \\
\left(\alpha-h_{1}+1\right) e_{9}-e_{5}
\end{array}\right\} \xi(k) \\
& =\left(\Pi_{1}+\Pi(h(k), \alpha)\right) \xi(k),
\end{aligned}
$$

it follows that

$$
\begin{aligned}
\Delta V_{1}(k) & \\
= & \xi^{T}(k)\left(\Pi_{1}+\Pi(h(k), \alpha)\right)^{T} P \\
& \times\left(\Pi_{1}+\Pi(h(k), \alpha)\right) \xi(k) \\
& -\xi^{T}(k)\left(\Pi_{2}+\Pi(h(k), \alpha)\right)^{T} P \\
& \times\left(\Pi_{2}+\Pi(h(k), \alpha)\right) \xi(k) \\
= & \xi^{T}(k)\left\{\begin{array}{c}
\Pi_{1}^{T} P \Pi_{1}-\Pi_{2}^{T} P \Pi_{2} \\
+2\left(\Pi_{1}-\Pi_{2}\right)^{T} P \Pi(h(k), \alpha)
\end{array}\right\} \xi(k) \\
= & \xi^{T}(k)\left(\Sigma_{11}+\Sigma_{12}(h(k), \alpha)\right) \xi(k) .
\end{aligned}
$$

The forward differences of $V_{2}(k)$ and $V_{3}(k)$ can be obtained as

$$
\begin{gathered}
\Delta V_{2}(k)=\sum_{j=k+1-h_{1}}^{k} x^{T}(j) Q_{1} x(j)-\sum_{j=k-h_{1}}^{k-1} x^{T}(j) \\
\cdot Q_{1} x(j)+\sum_{j=k+1-h_{2}}^{k-\alpha} x^{T}(j) Q_{2} x(j)-\sum_{j=k-h_{2}}^{k-\alpha-1} x^{T}(j) \\
\cdot Q_{2} x(j)+\sum_{j=k+1-\alpha}^{k-h_{1}} x^{T}(j) Q_{3} x(j)-\sum_{j=k-\alpha}^{k-h_{1}-1} x^{T}(j) \\
\cdot Q_{3} x(j)=x^{T}(k) Q_{1} x(k)-x^{T}\left(k-h_{1}\right) Q_{1} x(k \\
\left.-h_{1}\right)+x^{T}(k-\alpha) Q_{2} x(k-\alpha)-x^{T}\left(k-h_{2}\right)
\end{gathered}
$$$$
\text { - } Q_{2} x\left(k-h_{2}\right)+x^{T}\left(k-h_{1}\right) Q_{3} x\left(k-h_{1}\right)-x^{T}(k
$$$$
-\alpha) Q_{3} x(k-\alpha)=x^{T}(k) Q_{1} x(k)+x^{T}\left(k-h_{1}\right)
$$$$
\cdot\left(Q_{3}-Q_{1}\right) x\left(k-h_{1}\right)+x^{T}(k-\alpha)\left(Q_{2}-Q_{3}\right) x(k
$$$$
-\alpha)-x^{T}\left(k-h_{2}\right) Q_{2} x\left(k-h_{2}\right)=\xi^{T}(k)
$$ 
$\cdot\left\{\begin{array}{c}e_{1}^{T} Q_{1} e_{1}+e_{2}^{T}\left(Q_{3}-Q_{1}\right) e_{2} \\ +e_{5}^{T}\left(Q_{2}-Q_{3}\right) e_{5}-e_{4}^{T} Q_{2} e_{4}\end{array}\right\} \xi(k)=\xi^{T}(k)$

$\cdot \Sigma_{2} \xi(k)$,

$\Delta V_{3}(k)=\sum_{j=k+1-h(k+1)}^{k} \zeta_{2}^{T}(j) R \zeta_{2}(j)-\sum_{j=k-h(k)}^{k-1} \zeta_{2}^{T}(j)$

$\cdot R \zeta_{2}(j)+\sum_{i=-h_{2}+1}^{-h_{1}} \sum_{j=k+1+i}^{k} \zeta_{2}^{T}(j) R \zeta_{2}(j)$

$-\sum_{i=-h_{2}+1}^{-h_{1}} \sum_{j=k+i}^{k-1} \zeta_{2}^{T}(j) R \zeta_{2}(j)=\sum_{j=k+1-h(k+1)}^{k-h_{1}} \zeta_{2}^{T}(j)$

$\cdot R \zeta_{2}(j)+\sum_{j=k-h_{1}+1}^{k-1} \zeta_{2}^{T}(j) R \zeta_{2}(j)+\zeta_{2}^{T}(k) R \zeta_{2}(k)$

$-\sum_{j=k-h(k)}^{k-1} \zeta_{2}^{T}(j) R \zeta_{2}(j)$

$+\sum_{i=-h_{2}+1}^{-h_{1}}\left(\begin{array}{c}\sum_{j=k+i}^{k-1} \zeta_{2}^{T}(j) R \zeta_{2}(j)+\zeta_{2}^{T}(k) R \zeta_{2}(k) \\ -\zeta_{2}^{T}(k+i) R \zeta_{2}(k+i) \\ -\sum_{j=k+i}^{k-1} \zeta_{2}^{T}(j) R \zeta_{2}(j)\end{array}\right)$

$\leq \sum_{j=k+1-h_{2}}^{k-h_{1}} \zeta_{2}^{T}(j) R \zeta_{2}(j)+\sum_{j=k-h(k)+1}^{k-1} \zeta_{2}^{T}(j) R \zeta_{2}(j)$

$+\zeta_{2}^{T}(k) R \zeta_{2}(k)-\sum_{j=k-h(k)+1}^{k-1} \zeta_{2}^{T}(j) R \zeta_{2}(j)-\zeta_{2}^{T}(k$

$-h(k)) R \zeta_{2}(k-h(k))+\sum_{i=-h_{2}+1}^{-h_{1}} \zeta_{2}^{T}(k) R \zeta_{2}(k)$

$-\sum_{i=-h_{2}+1}^{-h_{1}} \zeta_{2}^{T}(k+i) R \zeta_{2}(k+i)=\sum_{j=k+1-h_{2}}^{k-h_{1}} \zeta_{2}^{T}(j)$

$\cdot R \zeta_{2}(j)+\zeta_{2}^{T}(k) R \zeta_{2}(k)-\zeta_{2}^{T}(k-h(k)) R \zeta_{2}(k$

$-h(k))+h_{12} \zeta_{2}^{T}(k) R \zeta_{2}(k)-\sum_{j=k+1-h_{2}}^{k-h_{1}} \zeta_{2}^{T}(j) R \zeta_{2}(j)$

$=\left(h_{12}+1\right) \zeta_{2}^{T}(k) R \zeta_{2}(k)-\zeta_{2}^{T}(k-h(k)) R \zeta_{2}(k$

$-h(k))=\xi^{T}(k)\left[\begin{array}{l}e_{1} \\ e_{10}\end{array}\right]^{T}\left(h_{12}+1\right) R\left[\begin{array}{l}e_{1} \\ e_{10}\end{array}\right] \xi(k)$
(27)

$$
-\xi^{T}(k)\left[\begin{array}{c}
e_{3} \\
e_{11}
\end{array}\right]^{T} R\left[\begin{array}{l}
e_{3} \\
e_{11}
\end{array}\right] \xi(k)=\xi^{T}(k) \Sigma_{3} \xi(k)
$$

$$
\begin{aligned}
& \Delta V_{4}(k)=h_{1} \sum_{i=-h_{1}}^{-1}\left(\begin{array}{c}
\sum_{j=k+1+i}^{k} \eta^{T}(j) Z_{1} \eta(j) \\
-\sum_{j=k+i}^{k-1} \eta^{T}(j) Z_{1} \eta(j)
\end{array}\right) \\
& +h_{12} \sum_{i=-h_{2}}^{-h_{1}-1}\left(\sum_{j=k+1+i}^{k} \eta^{T}(j) Z_{2} \eta(j)\right. \\
& \left.-\sum_{j=k+i}^{k-1} \eta^{T}(j) Z_{2} \eta(j)\right)=h_{1} \sum_{i=-h_{1}}^{-1}\left(\eta^{T}(k) Z_{1} \eta(k)\right. \\
& \left.-\eta^{T}(k+i) Z_{1} \eta(k+i)\right) \\
& +h_{12} \sum_{i=-h_{2}}^{-h_{1}-1}\left(\eta^{T}(k) Z_{2} \eta(k)-\eta^{T}(k+i) Z_{2} \eta(k+i)\right) \\
& =\eta^{T}(k)\left(h_{1}^{2} Z_{1}+h_{12}^{2} Z_{2}\right) \eta(k)-h_{1} \sum_{i=-h_{1}}^{-1} \eta^{T}(k+i) \\
& \cdot Z_{1} \eta(k+i)-h_{12} \sum_{i=-h_{2}}^{-h_{1}-1} \eta^{T}(k+i) Z_{2} \eta(k+i) \\
& =\xi^{T}(k)\left(e_{s}-e_{1}\right)^{T}\left(h_{1}^{2} Z_{1}+h_{12}^{2} Z_{2}\right)\left(e_{s}-e_{1}\right) \xi(k) \\
& -h_{1} \sum_{j=k-h_{1}}^{k-1} \eta^{T}(j) Z_{1} \eta(j)-h_{12} \sum_{j=k-h_{2}}^{k-h_{1}-1} \eta^{T}(j) Z_{2} \eta(j) .
\end{aligned}
$$

Using inequality (8) from Lemma 3 to estimate $Z_{1}$-dependent summation term yields

$$
\begin{aligned}
h_{1} & \sum_{j=k-h_{1}}^{k-1} \eta^{T}(j) Z_{1} \eta(j) \\
& \geq\left[\begin{array}{l}
\omega_{1} \\
\omega_{2}
\end{array}\right]^{T}\left[\begin{array}{cc}
Z_{1} & 0 \\
* & 3 \rho\left(0, h_{1}\right) Z_{1}
\end{array}\right]\left[\begin{array}{l}
\omega_{1} \\
\omega_{2}
\end{array}\right] \\
& =\xi^{T}(k) \Sigma_{42} \xi(k) .
\end{aligned}
$$

Similarly, by using (9) we estimate $Z_{2}$-dependent summation term

$$
\begin{aligned}
& h_{12} \sum_{j=k-h_{2}}^{k-h_{1}-1} \eta^{T}(j) Z_{2} \eta(j) \\
& =h_{12} \sum_{j=k-h_{2}}^{k-h(k)-1} \eta^{T}(j) Z_{2} \eta(j) \\
& \quad+h_{12} \sum_{j=k-h(k)}^{k-h_{1}-1} \eta^{T}(j) Z_{2} \eta(j)
\end{aligned}
$$




$$
\begin{aligned}
\geq & \frac{1}{\delta_{h}} \xi^{T}(k) E_{2}^{T}\left[\begin{array}{cc}
Z_{2} & 0 \\
0 & 3 Z_{2}
\end{array}\right] E_{2} \xi(k) \\
& +\frac{1}{1-\delta_{h}} \xi^{T}(k) E_{3}^{T}\left[\begin{array}{cc}
Z_{2} & 0 \\
0 & 3 Z_{2}
\end{array}\right] E_{3} \xi(k) \\
= & \xi^{T}(k)\left[\begin{array}{l}
E_{2} \\
E_{3}
\end{array}\right]^{T}\left[\begin{array}{cc}
\frac{1}{\delta_{h}} \widetilde{Z}_{2} & 0 \\
0 & \frac{1}{1-\delta_{h}} \widetilde{Z}_{2}
\end{array}\right]\left[\begin{array}{l}
E_{2} \\
E_{3}
\end{array}\right] \xi(k) .
\end{aligned}
$$

Based on Lemma 6, for any matrix $S \in \mathfrak{R}^{2 n \times 2 n}$ we have

$$
\begin{aligned}
& h_{12} \sum_{j=k-h_{2}}^{k-h_{1}-1} \eta^{T}(j) Z_{2} \eta(j) \geq \xi^{T}(k)\left[\begin{array}{l}
E_{2} \\
E_{3}
\end{array}\right]^{T} \\
& \cdot\left[\begin{array}{cc}
\widetilde{Z}_{2}+\left(1-\delta_{h}\right) T_{1} & S \\
* & \widetilde{Z}_{2}+\delta_{h} T_{2}
\end{array}\right]\left[\begin{array}{c}
E_{2} \\
E_{3}
\end{array}\right] \xi(k) \\
& =\xi^{T}(k) \Sigma_{43}(h(k)) \xi^{T}(k) .
\end{aligned}
$$

Then, we can get the upper bound of $\Delta V_{4}(k)$ as

$$
\begin{aligned}
\Delta V_{4}(k) & \leq \xi^{T}(k)\left\{\Sigma_{41}-\Sigma_{42}-\Sigma_{43}(h(k))\right\} \xi(k) \\
& =\xi^{T}(k) \Sigma_{4}(h(k)) \xi(k) .
\end{aligned}
$$

Calculating $\Delta V_{5}(k)$ gives

$$
\Delta V_{5}(k)
$$

$$
\begin{aligned}
& =\left(\alpha-h_{1}\right) \sum_{i=-\alpha}^{-h_{1}-1}\left(\begin{array}{c}
\sum_{j=k+i}^{k-1} \eta^{T}(j) Z_{3} \eta(j) \\
+\eta^{T}(k) Z_{3} \eta(k) \\
-\eta^{T}(k+i) Z_{3} \eta(k+i) \\
-\sum_{j=k+i}^{k-1} \eta^{T}(j) Z_{3} \eta(j)
\end{array}\right) \\
& =\left(\alpha-h_{1}\right)^{2} \eta^{T}(k) Z_{3} \eta(k) \\
& -\left(\alpha-h_{1}\right) \sum_{i=-\alpha}^{-h_{1}-1} \eta^{T}(k+i) Z_{3} \eta(k+i) \\
& =\xi^{T}(k)\left(e_{s}-e_{1}\right)^{T}\left(\alpha-h_{1}\right)^{2} Z_{3}\left(e_{s}-e_{1}\right) \xi(k) \\
& -\left(\alpha-h_{1}\right) \sum_{j=k-\alpha}^{k-h_{1}-1} \eta^{T}(j) Z_{3} \eta(j) .
\end{aligned}
$$

By inequality (8), the second term of $\Delta V_{5}(k)$ can be written as

$$
\begin{aligned}
& \left(\alpha-h_{1}\right) \sum_{j=k-\alpha}^{k-h_{1}-1} \eta^{T}(j) Z_{3} \eta(j) \\
& \geq\left[\begin{array}{l}
\omega_{3} \\
\omega_{4}
\end{array}\right]^{T}\left[\begin{array}{cc}
Z_{3} & 0 \\
* & 3 \rho\left(h_{1}, \alpha\right) Z_{3}
\end{array}\right]\left[\begin{array}{l}
\omega_{3} \\
\omega_{4}
\end{array}\right] .
\end{aligned}
$$

Then, we can get the upper bound of $\Delta V_{5}(k)$ as

$$
\Delta V_{5}(k)
$$

$$
\begin{aligned}
& \leq \xi^{T}(k)\left(\begin{array}{c}
\Sigma_{51}(\alpha) \\
-E_{4}^{T}\left[\begin{array}{cc}
Z_{3} & 0 \\
* & 3 \rho\left(h_{1}, \alpha\right) Z_{3}
\end{array}\right] E_{4}
\end{array}\right) \xi(k) \\
& =\xi^{T}(k)\left(\Sigma_{51}(\alpha)-\Sigma_{52}(\alpha)\right) \xi(k) .
\end{aligned}
$$

Under the assumption on the activation functions (5) and (6), for any positive definite diagonal matrices $M_{j}=$ $\operatorname{diag}\left\{m_{j 1}, m_{j 2}, \ldots, m_{j n}\right\}, j=1,2,3$, the following inequality holds:

$$
\begin{aligned}
& 0 \geq 2 \sum_{i=1}^{n} m_{1 i}\left[f_{i}\left(x_{i}(k)\right)-k_{i}^{-} x_{i}(k)\right] \\
& \times\left[f_{i}\left(x_{i}(k)\right)-k_{i}^{+} x_{i}(k)\right] \\
& +2 \sum_{i=1}^{n} m_{2 i}\left[f_{i}\left(x_{i}(k-h(k))\right)-k_{i}^{-} x_{i}(k-h(k))\right] \\
& \times\left[f_{i}\left(x_{i}(k-h(k))\right)-k_{i}^{+} x_{i}(k-h(k))\right] \\
& +2 \sum_{i=1}^{n} m_{3 i}\left[\begin{array}{c}
f_{i}\left(x_{i}(k)\right)-f_{i}\left(x_{i}(k-h(k))\right) \\
-k_{i}^{-}\left(x_{i}(k)-x_{i}(k-h(k))\right)
\end{array}\right] \\
& \times\left[\begin{array}{c}
f_{i}\left(x_{i}(k)\right)-f_{i}\left(x_{i}(k-h(k))\right) \\
-k_{i}^{+}\left(x_{i}(k)-x_{i}(k-h(k))\right)
\end{array}\right] .
\end{aligned}
$$

Then

$$
\begin{aligned}
0 \geq 2 & {\left[f(x(k))-K_{1} x(k)\right] M_{1}\left[f(x(k))-K_{2} x(k)\right] } \\
& +2\left[f(x(k-h(k)))-K_{1} x(k-h(k))\right] \\
& \times M_{2}\left[f(x(k-h(k)))-K_{2} x(k-h(k))\right] \\
& +2\left[\begin{array}{c}
f(x(k))-f(x(k-h(k))) \\
-K_{1}(x(k)-x(k-h(k)))
\end{array}\right] M_{3} \\
& \times\left[\begin{array}{c}
f(x(k))-f(x(k-h(k))) \\
-K_{2}(x(k)-x(k-h(k)))
\end{array}\right], \\
0 \geq & \xi^{T}(k)\left(e_{10}-K_{1} e_{1}\right)^{T} M_{1}\left(e_{10}-K_{2} e_{1}\right) \xi^{T}(k) \\
& +2 \xi^{T}(k)\left(e_{11}-K_{1} e_{3}\right)^{T} M_{2}\left(e_{11}-K_{2} e_{3}\right) \xi^{T}(k) \\
& +2 \xi^{T}(k)\left(e_{10}-e_{11}-K_{1}\left(e_{1}-e_{3}\right)\right)^{T} M_{3} \\
& \times\left(e_{10}-e_{11}-K_{2}\left(e_{1}-e_{3}\right)\right) \xi^{T}(k) .
\end{aligned}
$$

So, we have

$$
\xi^{T}(k) \Sigma_{S} \xi(k) \leq 0 .
$$

Therefore, by combining (26)-(28), (33), (36), and (40), the forward difference of $V(k)$ is obtained as

$$
\Delta V(k) \leq \xi^{T}(k) \sum(h(k), \alpha) \xi(k) .
$$


Since the matrix $\Sigma(h(k), \alpha)$ is affine with respect to the delay $h(k)$, the condition $\Sigma(h(k), \alpha)<0$ is satisfied if and only if

$$
\Sigma\left(h_{i}, \alpha\right)<0, \quad i=1,2 .
$$

By calculating, we have

$$
\begin{aligned}
\Sigma_{12}\left(h_{1}, \alpha\right)= & \operatorname{Sym}\left\{\left(\Pi_{1}-\Pi_{2}\right)^{T} P \Pi\left(h_{1}, \alpha\right)\right\}, \\
\Sigma_{12}\left(h_{2}, \alpha\right)= & \operatorname{Sym}\left\{\left(\Pi_{1}-\Pi_{2}\right)^{T} P \Pi\left(h_{2}, \alpha\right)\right\}, \\
\Sigma_{43}\left(h_{1}\right)= & {\left[\begin{array}{l}
E_{2} \\
E_{3}
\end{array}\right]^{T}\left(\left[\begin{array}{cc}
\widetilde{Z}_{2} & S \\
* & \widetilde{Z}_{2}
\end{array}\right]+\left[\begin{array}{ll}
T_{1} & 0 \\
* & 0
\end{array}\right]\right)\left[\begin{array}{l}
E_{2} \\
E_{3}
\end{array}\right] } \\
= & {\left[\begin{array}{l}
E_{2} \\
E_{3}
\end{array}\right]^{T}\left[\begin{array}{cc}
\widetilde{Z}_{2} & S \\
* & \widetilde{Z}_{2}
\end{array}\right]\left[\begin{array}{l}
E_{2} \\
E_{3}
\end{array}\right]+E_{2}^{T} \widetilde{Z}_{2} E_{2} } \\
& -E_{2}^{T} S \widetilde{Z}_{2}^{-1} S^{T} E_{2} \\
= & \widetilde{\Sigma}_{43}\left(h_{1}\right)-E_{2}^{T} S \widetilde{Z}_{2}^{-1} S^{T} E_{2}, \\
\Sigma_{43}\left(h_{2}\right)= & {\left[\begin{array}{l}
E_{2} \\
E_{3}
\end{array}\right]^{T}\left(\left[\begin{array}{ll}
\widetilde{Z}_{2} & S \\
* & \widetilde{Z}_{2}
\end{array}\right]+\left[\begin{array}{ll}
0 & 0 \\
* & T_{2}
\end{array}\right]\right)\left[\begin{array}{l}
E_{2} \\
E_{3}
\end{array}\right] } \\
= & {\left[\begin{array}{l}
E_{2} \\
E_{3}
\end{array}\right]^{T}\left[\begin{array}{ll}
\widetilde{Z}_{2} & S \\
* & \widetilde{Z}_{2}
\end{array}\right]\left[\begin{array}{l}
E_{2} \\
E_{3}
\end{array}\right]+E_{3}^{T} \widetilde{Z}_{2} E_{3} } \\
& -E_{3}^{T} S^{T} \widetilde{Z}_{2}^{-1} S E_{3} \\
= & \widetilde{\Sigma}_{43}\left(h_{2}\right)-E_{3}^{T} S^{T} \widetilde{Z}_{2}^{-1} S E_{3}, \\
\Sigma\left(h_{1}, \alpha\right)= & \Phi\left(h_{1}, \alpha\right)+E_{2}^{T} S \widetilde{Z}_{2}^{-1} S^{T} E_{2}<0, \\
\Sigma\left(h_{2}, \alpha\right)= & \Phi\left(h_{2}, \alpha\right)+E_{3}^{T} S^{T} \widetilde{Z}_{2}^{-1} S E_{3}<0 .
\end{aligned}
$$

Then, by taking Schur complement, it can be seen that (46) and (47) are equivalent to (18) and (19), respectively. Therefore, when (18) and (19) hold, $\Delta V(k)<0$, which shows that system (4) is asymptotically stable.

Remark 11. In Theorem 10, the Wirtinger-based summation inequality (8) is applied to summation with the constant lower and upper bound $\left(h_{1} \sum_{j=k-h_{1}}^{k-1} \eta^{T}(j) Z_{1} \eta(j)\right)$. However, in the case of the summation with the time-varying lower or upper bound

$$
h_{12} \sum_{j=k-h_{2}}^{k-h(k)-1} \eta^{T}(j) Z_{2} \eta(j)+h_{12} \sum_{j=k-h(k)}^{k-h_{1}-1} \eta^{T}(j) Z_{2} \eta(j)
$$

combination of the Wirtinger-based inequality (9) and the reciprocally convex approach (13) is applied.

By introducing an augmented LKF and zero equations, a further improved stability condition of system (4) can be obtained as follows.

Theorem 12. For given positive integers $h_{1}$ and $h_{2}$, system (4) with interval time-varying delay satisfying condition (2) is asymptotically stable, if there exist positive-definite matrices $P \in \Re^{4 n \times 4 n}, Q_{i} \in \Re^{n \times n}, i=1,2,3, R \in \Re^{2 n \times 2 n}, Z_{i} \in \Re^{n \times n}$, $i=1,2,3, G \in \Re^{2 n \times 2 n}$, positive definite diagonal matrices $M_{i} \in \mathfrak{R}^{n \times n}, i=1,2,3$, symmetric matrices $H_{i} \in \mathfrak{R}^{n \times n}, i=1,2$, and any matrices $S \in \mathfrak{R}^{2 n \times 2 n}$ and $X \in \mathfrak{R}^{2 n \times 2 n}$ such that the following LMIs hold:

$$
\begin{gathered}
{\left[\begin{array}{cc}
G_{1} & X \\
* & G_{2}
\end{array}\right]>0,} \\
{\left[\begin{array}{cc}
\Phi\left(h_{1}, \alpha\right)+\Sigma_{61}-\Sigma_{62}\left(h_{1}\right) & E_{2}^{T} S \\
* & -\widetilde{Z}_{2}
\end{array}\right]<0,} \\
{\left[\begin{array}{cc}
\Phi\left(h_{2}, \alpha\right)+\Sigma_{61}-\Sigma_{62}\left(h_{2}\right) & E_{3}^{T} S^{T} \\
* & -\widetilde{Z}_{2}
\end{array}\right]<0,}
\end{gathered}
$$

where

$$
\begin{gathered}
\Phi\left(h_{i}, \alpha\right)=\Sigma_{11}+\Sigma_{12}\left(h_{i}, \alpha\right)+\Sigma_{2}+\Sigma_{3}+\Sigma_{41}-\Sigma_{42} \\
-\widetilde{\Sigma}_{43}\left(h_{i}\right)+\Sigma_{51}(\alpha)-\Sigma_{52}(\alpha)-\Sigma_{s}, \\
i=1,2 .
\end{gathered}
$$

Proof. Construct the following LKF candidate:

$$
\widetilde{V}(k)=V(k)+V_{6}(k),
$$

where

$$
V_{6}(k)=h_{12} \sum_{i=-h_{2}}^{-h_{1}-1} \sum_{j=k+i}^{k-1} \zeta_{3}^{T}(j) G \xi_{3}(j) .
$$

The forward difference of (54) along the trajectories of the DNN (4) gives

$$
\begin{aligned}
\Delta V_{6}(k)= & h_{12}^{2} \zeta_{3}^{T}(k) G \xi_{3}(k) \\
& -h_{12} \sum_{j=k-h_{2}}^{k-h(k)-1} \zeta_{3}^{T}(j) G \xi_{3}(j) \\
& -h_{12} \sum_{j=k-h(k)}^{k-h_{1}-1} \zeta_{3}^{T}(j) G \xi_{3}(j) .
\end{aligned}
$$

For any symmetric matrices $H_{i}, i=1,2$, we have

$$
\begin{aligned}
& \sum_{j=k-l_{2}}^{k-l_{1}-1}\left(x^{T}(j+1) H_{i} x(j+1)-x^{T}(j) H_{i} x(j)\right) \\
& =x^{T}\left(k-l_{1}\right) H_{i} x\left(k-l_{1}\right) \\
& \quad-x^{T}\left(k-l_{2}\right) H_{i} x\left(k-l_{2}\right) .
\end{aligned}
$$


Since

$$
\begin{aligned}
x^{T}( & +1) H_{i} x(j+1)-x^{T}(j) H_{i} x(j) \\
= & (x(j)+\eta(k))^{T} H_{i}(x(j)+\eta(k)) \\
& -x^{T}(j) H_{i} x(j) \\
= & \eta^{T}(j) H_{i} \eta(j)+2 x^{T}(j) H_{i} \eta(j) \\
= & {\left[\begin{array}{l}
x(j) \\
\eta(j)
\end{array}\right]^{T}\left[\begin{array}{ll}
0 & H_{i} \\
* & H_{i}
\end{array}\right]\left[\begin{array}{l}
x(j) \\
\eta(j)
\end{array}\right] } \\
= & \zeta_{3}^{T}(k)\left[\begin{array}{ll}
0 & H_{i} \\
* & H_{i}
\end{array}\right] \xi_{3}(j),
\end{aligned}
$$

then the following zero equation holds:

$$
\begin{aligned}
E Q\left(l_{1}, l_{2}, H_{i}\right)= & x^{T}\left(k-l_{1}\right) H_{i} x\left(k-l_{1}\right) \\
& -x^{T}\left(k-l_{2}\right) H_{i} x\left(k-l_{2}\right) \\
& -\sum_{j=k-l_{2}}^{k-l_{1}-1} \zeta_{3}^{T}(k)\left[\begin{array}{cc}
0 & H_{i} \\
* & H_{i}
\end{array}\right] \xi_{3}(j)=0 .
\end{aligned}
$$

Now, from (58) we get the following two-zero equations:

$$
\begin{aligned}
\operatorname{EQ}\left(h_{1}, h(k), H_{1}\right)= & x^{T}\left(k-h_{1}\right) H_{1} x\left(k-h_{1}\right) \\
& -x^{T}(k-h(k)) H_{1} x(k-h(k)) \\
& -\sum_{j=k-h(k)}^{k-h_{1}-1} \zeta_{3}^{T}(k)\left[\begin{array}{cc}
0 & H_{1} \\
* & H_{1}
\end{array}\right] \xi_{3}(j) \\
= & 0,
\end{aligned}
$$$$
\operatorname{EQ}\left(h(k), h_{2}, H_{2}\right)=x^{T}(k-h(k)) H_{2} x(k-h(k))
$$$$
-x^{T}\left(k-h_{2}\right) H_{2} x\left(k-h_{2}\right)
$$$$
-\sum_{j=k-h_{2}}^{k-h(k)-1} \zeta_{3}^{T}(k)\left[\begin{array}{ll}
0 & H_{2} \\
* & H_{2}
\end{array}\right] \xi_{3}(j)
$$$$
=0 \text {. }
$$

Adding the inequalities (59) and (60) to (55) implies

$$
\begin{aligned}
\Delta V_{6}(k)= & h_{12}^{2} \zeta_{3}^{T}(k) G \xi_{3}(k) \\
& +h_{12} x^{T}\left(k-h_{1}\right) H_{1} x\left(k-h_{1}\right) \\
& -h_{12} x^{T}(k-h(k))\left(H_{1}-H_{2}\right) x(k-h(k)) \\
& -h_{12} x^{T}\left(k-h_{2}\right) H_{2} x\left(k-h_{2}\right)
\end{aligned}
$$

$$
\begin{aligned}
& -h_{12} \sum_{j=k-h(k)}^{k-h_{1}-1} \zeta_{3}^{T}(j) G_{1} \xi_{3}(j) \\
& -h_{12} \sum_{j=k-h_{2}}^{k-h(k)-1} \zeta_{3}^{T}(j) G_{2} \xi_{3}(j) .
\end{aligned}
$$

Applying Jensen inequality (7), $\Delta V_{6}(k)$ is estimated as $\Delta V_{6}(k)$

$$
\leq \xi^{T}(k)\left\{\begin{array}{c}
h_{12}^{2}\left[\begin{array}{c}
e_{1} \\
e_{s}-e_{1}
\end{array}\right]^{T} G\left[\begin{array}{c}
e_{1} \\
e_{s}-e_{1}
\end{array}\right] \\
+h_{12} e_{2}^{T} H_{1} e_{2}-h_{12} e_{4}^{T} H_{2} e_{4} \\
-h_{12} e_{3}^{T}\left(H_{1}-H_{2}\right) e_{3}
\end{array}\right\} \xi(k)
$$

$$
\begin{aligned}
& -\frac{1}{\delta_{h}} \sum_{j=k-h(k)}^{k-h_{1}-1} \zeta_{3}^{T}(j) G_{1} \sum_{j=k-h(k)}^{k-h_{1}-1} \xi_{3}(j) \\
& -\frac{1}{1-\delta_{h}} \sum_{j=k-h_{2}}^{k-h(k)-1} \zeta_{3}^{T}(j) G_{2} \sum_{j=k-h_{2}}^{k-h(k)-1} \xi_{3}(j) \\
= & \xi^{T}(k) \Sigma_{61} \xi(k) \\
& -\frac{1}{\delta_{h}} \xi^{T}(k) E_{5}^{T}(h(k)) G_{1} E_{5}(h(k)) \xi(k) \\
& -\frac{1}{1-\delta_{h}} \xi^{T}(k) E_{6}^{T}(h(k)) G_{2} E_{6}(h(k)) \xi(k) .
\end{aligned}
$$

Using Lemma 5, for any matrix $X \in \mathfrak{R}^{2 n \times 2 n}$ we have

$$
\begin{aligned}
& \Delta V_{6}(k) \leq \xi^{T}(k) \\
& \cdot\left(\Sigma_{61}-E_{56}^{T}(h(k))\left[\begin{array}{cc}
G_{1} & X \\
* & G_{2}
\end{array}\right] E_{56}(h(k))\right) \xi(k) \\
& =\xi^{T}(k)\left(\Sigma_{61}-E_{56}^{T}(h(k)) G_{12} E_{56}(h(k))\right) \xi(k) \\
& =\xi^{T}(k)\left[\Sigma_{61}-\Sigma_{62}(h(k))\right] \xi(k)=\xi^{T}(k) \Sigma_{6}(h(k)) \\
& \cdot \xi(k) .
\end{aligned}
$$

Finally

$\Delta \widetilde{V}(k)$

$$
\leq \xi^{T}(k)\left[\Sigma(h(k), \alpha)+\Sigma_{61}-\Sigma_{62}(h(k))\right] \xi(k) .
$$

Based on Lemma 9, the equivalent condition of $\Sigma(h(k), \alpha)+$ $\Sigma_{61}-E_{56}^{T}(h(k)) G_{12} E_{56}(h(k))<0$ is that matrix $\Psi$ exists such that

$$
\Theta(h(k), \alpha)=\left[\begin{array}{c|c}
\Sigma(h(k), \alpha)+\Sigma_{61} & \Psi^{T} \\
+\operatorname{Sym}\left\{E_{56}^{T}(h(k)) \Psi\right\} & \\
\hline * & -G_{12}
\end{array}\right]
$$


TABLE 1: MAUB $h_{2 \max }$ for given $h_{1}$ in Example 1.

\begin{tabular}{|c|c|c|c|c|c|c|c|c|}
\hline \multirow{2}{*}{ Method } & \multicolumn{7}{|c|}{$h_{1}$} & \multirow{2}{*}{ NDVs } \\
\hline & 2 & 4 & 6 & 8 & 10 & 15 & 20 & \\
\hline Th. $2[40]$ & 19 & 19 & 20 & 21 & 22 & 24 & 27 & $17.5 n^{2}+8.5 n$ \\
\hline Th. 1 [40] & 20 & 20 & 21 & 21 & 22 & 24 & 27 & $53.5 n^{2}+8.5 n$ \\
\hline Th. 1 [31] & - & 20 & 20 & 21 & 22 & 24 & - & $13.5 n^{2}+11.5 n$ \\
\hline Th. $3.1[24]$ & 19 & 19 & 20 & 20 & 21 & 24 & 27 & $11 n^{2}+6 n$ \\
\hline Cor. 2 [41] & 19 & 20 & 20 & 21 & 21 & 24 & 27 & $154 n^{2}+6 n$ \\
\hline Theorem 10 & 19 & 20 & 20 & 21 & 22 & 24 & 27 & $17 n^{2}+9 n$ \\
\hline Theorem 12 & 20 & 20 & 21 & 21 & 22 & 24 & 27 & $23 n^{2}+11 n$ \\
\hline
\end{tabular}

Since the matrix $\Theta(h(k), \alpha)$ is affine with respect to the delay $h(k)$, the condition $\Theta(h(k), \alpha)<0$ is satisfied if and only if

$$
\Theta\left(h_{i}, \alpha\right)<0, \quad i=1,2 .
$$

Based on Lemma 9, (66) is equivalent to

$$
\begin{aligned}
& \sum\left(h_{i}, \alpha\right)+\Sigma_{61}-E_{56}^{T}\left(h_{i}\right) G_{12} E_{56}\left(h_{i}\right) \\
& \quad=\Sigma\left(h_{i}, \alpha\right)+\Sigma_{61}-\Sigma_{62}\left(h_{i}\right)<0, \quad i=1,2,
\end{aligned}
$$

i.e.,

$$
\begin{aligned}
& \Phi\left(h_{1}, \alpha\right)+E_{2}^{T} S \widetilde{Z}_{2}^{-1} S^{T} E_{2}+\Sigma_{61}-\Sigma_{62}\left(h_{1}\right)<0 \\
& \Phi\left(h_{2}, \alpha\right)+E_{3}^{T} S^{T} \widetilde{Z}_{2}^{-1} S E_{3}+\Sigma_{61}-\Sigma_{62}\left(h_{2}\right)<0 .
\end{aligned}
$$

Then, by taking Schur complement, it can be seen that (68) and (69) are equivalent to (50) and (51), respectively. Therefore, when (50) and (51) hold, $\Delta \widetilde{V}(k)<0$, which shows that system (4) is asymptotically stable.

Remark 13. In order to improve the stability criterion proposed in Theorem 10, a new double summation term $V_{6}(k)$ with vector $\xi_{3}(k)=\left[x^{T}(k) \eta^{T}(k)\right]^{T}$ and free matrix $G$ is introduced in LKF. Further, in the proof of Theorem 12, two zero equalities with symmetric matrices $H_{1}$ and $H_{2}$ are introduced in $\Delta V_{6}(k)$. In this way, the conservatism of the criterion is reduced.

Remark 14. In order to reduce the number of decision variables, Lemma 9 is used twice. First, by using the lemma, nonaffine term

$$
\sum(h(k), \alpha)+\Sigma_{61}-E_{56}^{T}(h(k)) G_{12} E_{56}(h(k))<0
$$

with respect to $h(k)$ is transformed into the affine term $\Theta(h(k), \alpha)$. On that occasion, additional matrix $\Psi \in \mathfrak{R}^{11 n \times 2 n}$ is introduced in (65) and the number of decision variables has increased significantly (for $22 n^{2}$ ). Second, if we apply Lemma 9 on (66), then the matrix $\Psi \in \mathfrak{R}^{11 n \times 2 n}$ can be eliminated from $\Theta\left(h_{i}, \alpha\right)$. In this way, the significant reduction of decision variables was performed.

Remark 15. The proposed stability criterion depends on the lower and upper bounds of time-delay, $h_{1}$ and $h_{2}$. In order to compare our results with existing ones, a maximum allowable upper bound (MAUB) of the time-delay, $h_{2 \max }$, is adopted, such that the concerned system is asymptotically stable for any delay size less than the MAUB. Note that a criterion that gives a lower value of MAUB is less conservative with respect to other criteria.

\section{Demonstrative Examples}

In the section bellow, we provide three numerical examples to illustrate the effectiveness of the stability criteria proposed in this paper.

Example 1. Consider the DNN (4) with the following parameters:

$$
\begin{aligned}
C & =\left[\begin{array}{cc}
0.8 & 0 \\
0 & 0.9
\end{array}\right], \\
A & =\left[\begin{array}{cc}
0.001 & 0 \\
0 & 0.005
\end{array}\right], \\
A_{d} & =\left[\begin{array}{cc}
-0.1 & 0.01 \\
-0.2 & -0.1
\end{array}\right],
\end{aligned}
$$

which was used to check the feasible region of stability criteria in $[24,31,40,41]$. The activation function is in the form of $f(x)=\tanh (x)$ and satisfies Assumption 1 with $k_{1}^{-}=k_{2}^{-}=0$ and $k_{1}^{+}=k_{2}^{+}=1$. By using Theorem 10, the MAUB $h_{2 \max }$ is computed with different lover bounds $h_{1}$ and the obtained results are given in Table 1 . The number of decision variables (NDVs) is also given to show computation complexity. From this table, we can see that our results are less conservative and/or have less decision variables than [24, 31, 40, 41].

The time-varying delay and the state trajectories of the DNN (4) with parameters (71) for initial value $\phi(j)=\left[\begin{array}{ll}3 & 1\end{array}\right]^{T}$, $j \in\{-22,-21, \ldots,-1,0\}$ are shown in Figures 1 and 2 , which shows that the DNN (4) is asymptotically stable for $10 \leq$ $h(k) \leq 22$.

Example 2. Consider the DNN (4) with the following parameters [31]:

$$
C=\left[\begin{array}{cc}
0.1 & 0 \\
0 & 0.3
\end{array}\right] \text {, }
$$


TABLE 2: MAUB $h_{2 \max }$ for given $h_{1}$ in Example 2.

\begin{tabular}{lccccccc}
\hline Method & & \multicolumn{3}{c}{$h_{1}$} & & & \\
& 2 & 4 & 6 & 8 & 10 & 20 & 117 \\
\hline Th. $1[31]$ & 99 & 101 & 103 & 105 & 107 & $13.5 n^{2}+11.5 n$ \\
\hline Theorem 10 & 2028 & 2030 & 2032 & 2034 & 2036 & 2046 & $17 n^{2}+9 n$ \\
\hline Theorem 12 & 3140 & 3142 & 3144 & 3146 & 3148 & 3158 & $23 n^{2}+11 n$ \\
\hline
\end{tabular}

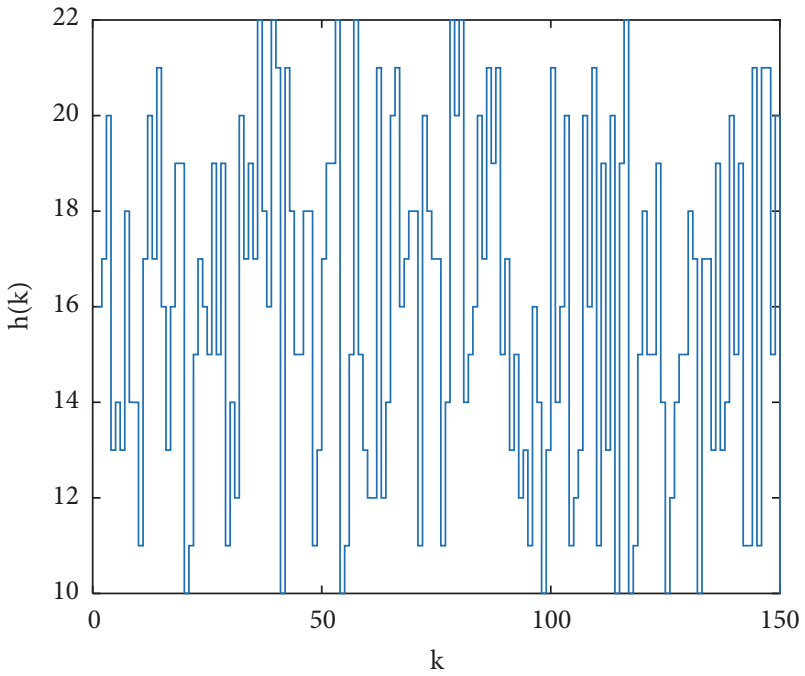

FIgURE 1: The time-varying delay.

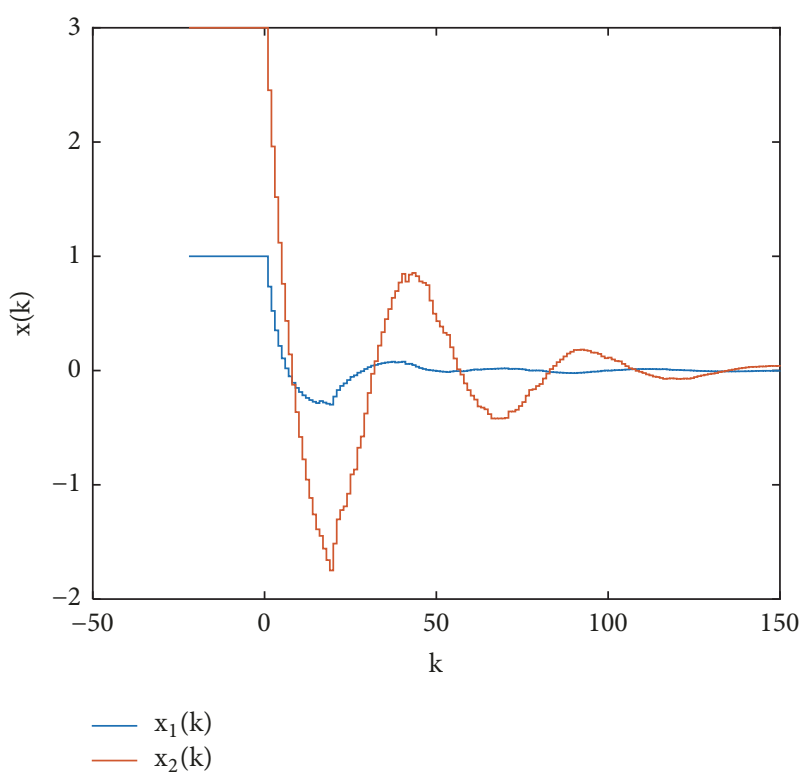

FIGURE 2: State trajectories of the DNN.

$$
\begin{aligned}
A & =\left[\begin{array}{cc}
0.02 & 0 \\
0 & 0.004
\end{array}\right], \\
A_{d} & =\left[\begin{array}{cc}
-0.01 & 0.01 \\
-0.02 & -0.01
\end{array}\right],
\end{aligned}
$$

and activation functions that satisfy Assumption 1 with $k_{1}^{-}=$ $k_{2}^{-}=0$ and $k_{1}^{+}=k_{2}^{+}=1$. In this example, the MAUB $h_{2 \max }$ is computed by using Theorems 10 and 12 for different lover bounds $h_{1}$ and results are shown in Table 2. From this table, it can be seen that the proposed stability criteria, Theorems 10 and 12, provide significantly larger MAUB than the previous result [31], while the numbers of decision variables are slightly higher.

Example 3. Consider the DNN (4) with the following [40]:

$$
\begin{aligned}
& C=\left[\begin{array}{ccc}
0.4 & 0 & 0 \\
0 & 0.3 & 0 \\
0 & 0 & 0.3
\end{array}\right], \\
& A=\left[\begin{array}{ccc}
0.2 & -0.2 & 0.1 \\
0 & -0.3 & 0.3 \\
-0.2 & -0.1 & -0.2
\end{array}\right], \\
& A_{d}=\left[\begin{array}{ccc}
-0.2 & 0.1 & 0 \\
-0.2 & 0.3 & 0.1 \\
0.1 & -0.2 & 0.3
\end{array}\right], \\
& k_{1}^{-}=0, \\
& k_{2}^{-}=-0.4, \\
& k_{3}^{-}=-0.2, \\
& k_{1}^{+}=0.6 \\
& k_{2}^{+}=0 \\
& k_{3}^{+}=0 .
\end{aligned}
$$

By applying Theorems 10 and 12 to the DNN (4) with parameters (73), the MAUB $h_{2 \max }$ is computed for different lover bounds $h_{1}$ and results are shown in Table 3. From this table it can be seen that stability criterion proposed in Theorem 12 gives approximately the same results but with significantly smaller number of decision variables than [40].

\section{Conclusion}

In this study, a novel Lyapunov-Krasovskii functional with single and double summation terms and several augmented vectors is constructed by decomposing the time-delay interval into two nonequidistant subintervals. Using Wirtingerbased inequality, extended reciprocally convex approach, and 
TABLE 3: MAUB $h_{2 \max }$ for given $h_{1}$ in Example 3.

\begin{tabular}{|c|c|c|c|c|c|c|c|}
\hline \multirow{2}{*}{ Method } & \multicolumn{6}{|c|}{$h_{1}$} & \multirow{2}{*}{ NDVs } \\
\hline & 2 & 4 & 6 & 10 & 15 & 20 & \\
\hline Th. 2 [40] & 13 & 15 & 17 & 21 & 26 & 31 & $17.5 n^{2}+8.5 n$ \\
\hline Th. 1 [40] & 18 & 20 & 22 & 26 & 31 & 36 & $53.5 n^{2}+8.5 n$ \\
\hline Theorem 10 & 13 & 15 & 16 & 19 & 24 & 29 & $17 n^{2}+9 n$ \\
\hline Theorem 12 & 19 & 20 & 22 & 26 & 31 & 36 & $23 n^{2}+11 n$ \\
\hline
\end{tabular}

several zero equalities, new stability conditions are developed in the form of linear matrix inequalities. It is shown that the proposed results are less conservative than the existing ones. Several numerical examples are presented to show the effectiveness and less conservativeness of the proposed method.

\section{Data Availability}

The [tables and figures] data used to support the findings of this study are included within the article.

\section{Conflicts of Interest}

The authors declare that there are no conflicts of interest regarding the publication of this paper.

\section{Acknowledgments}

This work was also supported in part by the Ministry of Science and Technological Development of Republic of Serbia under Grant ON174001.

\section{References}

[1] Y.-H. Chen and S.-C. Fang, "Neurocomputing with time delay analysis for solving convex quadratic programming problems," IEEE Transactions on Neural Networks and Learning Systems, vol. 11, no. 1, pp. 230-240, 2000.

[2] L. Zhang and Z. Yi, "Selectable and unselectable sets of neurons in recurrent neural networks with saturated piecewise linear transfer function," IEEE Transactions on Neural Networks and Learning Systems, vol. 22, no. 7, pp. 1021-1031, 2011.

[3] Y. Liu, Z. Wang, and X. Liu, "Global exponential stability of generalized recurrent neural networks with discrete and distributed delays," Neural Networks, vol. 19, no. 5, pp. 667-675, 2006.

[4] J. Sun, G. P. Liu, J. Chen, and D. Rees, "Improved delay-rangedependent stability criteria for linear systems with time-varying delays," Automatica, vol. 46, no. 2, pp. 466-470, 2010.

[5] O. M. Kwon, M. J. Park, J. H. Park, S. M. Lee, and E. J. Cha, "Improved delay-partitioning approach to robust stability analysis for discrete-time systems with time-varying delays and randomly occurring parameter uncertainties," Optimal Control Applications and Methods, vol. 36, no. 4, pp. 496-511, 2015.

[6] Y. He, M.-D. Ji, C.-K. Zhang, and M. Wu, "Global exponential stability of neural networks with time-varying delay based on free-matrix-based integral inequality," Neural Networks, vol. 77, pp. 80-86, 2016.
[7] H. Chen, S. Zhong, and J. Yang, "A New globally exponential stability criterion for neural networks with discrete and distributed delays," Mathematical Problems in Engineering, vol. 2015, Article ID 807150, 9 pages, 2015.

[8] S.-P. Xiao, H.-H. Lian, H.-B. Zeng, G. Chen, and W.-H. Zheng, "Analysis on robust passivity of uncertain neural networks with time-varying delays via free-matrix-based integral inequality," International Journal of Control, Automation, and Systems, vol. 15, no. 5, pp. 2385-2394, 2017.

[9] X.-M. Zhang, Q.-L. Han, X. Ge, and D. Ding, "An overview of recent developments in Lyapunov-Krasovskii functionals and stability criteria for recurrent neural networks with timevarying delays," Neurocomputing, vol. 313, pp. 392-401, 2018.

[10] H. G. Zhang, Z. S. Wang, and D. R. Liu, "A comprehensive review of stability analysis of continuous-time recurrent neural networks," IEEE Transactions on Neural Networks and Learning Systems, vol. 25, no. 7, pp. 1229-1262, 2014.

[11] C. Song, H. Gao, and W. X. Zheng, "A new approach to stability analysis of discrete-time recurrent neural networks with timevarying delay," Neurocomputing, vol. 72, no. 10-12, pp. 25632568, 2009.

[12] D. Zhang, L. Yu, Q.-G. Wang, and C.-J. Ong, "Estimator design for discrete-time switched neural networks with asynchronous switching and time-varying delay," IEEE Transactions on Neural Networks and Learning Systems, vol. 23, no. 5, pp. 827-834, 2012.

[13] O. M. Kwon, M. J. Park, J. H. Park, S. M. Lee, and E. J. Cha, "Stability and stabilization for discrete-time systems with timevarying delays via augmented Lyapunov-Krasovskii functional," Journal of The Franklin Institute, vol. 350, no. 3, pp. 521-540, 2013.

[14] L. Hou and H. Zhu, "Stability of stochastic discrete-time neural networks with discrete delays and the leakage delay," Mathematical Problems in Engineering, vol. 2015, Article ID 306806, 13 pages, 2015.

[15] Y. Li, "Exponential stability results of discrete-time stochastic neural networks with time-varying delays," Mathematical Problems in Engineering, vol. 2013, Article ID 486257, 10 pages, 2013.

[16] R. Saravanakumar, S. B. Stojanovic, D. D. Radosavljevic, C. K. Ahn, and H. R. Karimi, "Finite-time passivity-based stability criteria for delayed discrete-time neural networks via new weighted summation inequalities," IEEE Transactions on Neural Networks and Learning Systems, pp. 1-14, 2018.

[17] D.-H. Lin, J. Wu, and J.-N. Li, "Less conservative stability condition for uncertain discrete-time recurrent neural networks with time-varying delays," Neurocomputing, vol. 173, pp. 1578-1588, 2016.

[18] M. Wu, F. Liu, P. Shi, Y. He, and R. Yokoyama, "Improved freeweighting matrix approach for stability analysis of discretetime recurrent neural networks with time-varying delay," IEEE Transactions on Circuits and Systems II: Express Briefs, vol. 55, no. 7, pp. 690-694, 2008. 
[19] T. Wang, M. Xue, S. Fei, and T. Li, "Triple Lyapunov functional technique on delay-dependent stability for discrete-time dynamical networks," Neurocomputing, vol. 122, pp. 221-228, 2013.

[20] X.-M. Zhang, Q.-L. Han, A. Seuret, and F. Gouaisbaut, "An improved reciprocally convex inequality and an augmented Lyapunov-Krasovskii functional for stability of linear systems with time-varying delay," Automatica, vol. 84, pp. 221-226, 2017.

[21] O. M. Kwon, M. J. Park, J. H. Park, S. M. Lee, and E. J. Cha, "On stability analysis for neural networks with interval timevarying delays via some new augmented Lyapunov-Krasovskii functional," Communications in Nonlinear Science and Numerical Simulation, vol. 19, no. 9, pp. 3184-3201, 2014.

[22] M. S. Ali, R. Saravanakumar, and S. Arik, "Novel H state estimation of static neural networks with interval time-varying delays via augmented Lyapunov-Krasovskii functional," Neurocomputing, vol. 171, pp. 949-954, 2016.

[23] C.-K. Zhang, Y. He, L. Jiang, W.-J. Lin, and M. Wu, "Delaydependent stability analysis of neural networks with timevarying delay: a generalized free-weighting-matrix approach," Applied Mathematics and Computation, vol. 294, pp. 102-120, 2017.

[24] X.-G. Liu, F.-X. Wang, and Y.-J. Shu, "A novel summation inequality for stability analysis of discrete-time neural networks," Journal of Computational and Applied Mathematics, vol. 304, pp. 160-171, 2016.

[25] A. Seuret, F. Gouaisbaut, and E. Fridman, "Stability of discretetime systems with time-varying delays via a novel summation inequality," Institute of Electrical and Electronics Engineers Transactions on Automatic Control, vol. 60, no. 10, pp. 27402745, 2015.

[26] C.-K. Zhang, Y. He, L. Jiang, and M. Wu, "An improved summation inequality to discrete-time systems with timevarying delay," Automatica, vol. 74, pp. 10-15, 2016.

[27] S. Xiao, L. Xu, H. Zeng, and K. L. Teo, "Improved Stability Criteria for Discrete-time Delay Systems via Novel Summation Inequalities," International Journal of Control, Automation, and Systems, vol. 16, no. 4, pp. 1592-1602, 2018.

[28] X.-M. Zhang and Q.-L. Han, "New Lyapunov-Krasovskii functionals for global asymptotic stability of delayed neural networks," IEEE Transactions on Neural Networks and Learning Systems, vol. 20, no. 3, pp. 533-539, 2009.

[29] C. Ge, C. Hua, and X. Guan, "New delay-dependent stability criteria for neural networks with time-varying delay using delay-decomposition approach," IEEE Transactions on Neural Networks and Learning Systems, vol. 25, no. 7, pp. 1378-1383, 2014.

[30] P. G. Park, J. W. Ko, and C. Jeong, "Reciprocally convex approach to stability of systems with time-varying delays," Automatica, vol. 47, no. 1, pp. 235-238, 2011.

[31] C.-K. Zhang, Y. He, L. Jiang, Q.-G. Wang, and M. Wu, "Stability analysis of discrete-time neural networks with time-varying delay via an extended reciprocally convex matrix inequality," IEEE Transactions on Cybernetics, vol. 47, no. 10, pp. 3040-3049, 2017.

[32] L. Jarina Banu, P. Balasubramaniam, and K. Ratnavelu, "Robust stability analysis for discrete-time uncertain neural networks with leakage time-varying delay," Neurocomputing, vol. 151, no. 2, pp. 808-816, 2015.

[33] O. M. Kwon, M. J. Park, J. H. Park, S. M. Lee, and E. J. Cha, "New criteria on delay-dependent stability for discrete-time neural networks with time-varying delays," Neurocomputing, vol. 121, pp. 185-194, 2013.

[34] P. T. Nam, P. N. Pathirana, and H. Trinh, "Discrete Wirtingerbased inequality and its application," Journal of The Franklin Institute, vol. 352, no. 5, pp. 1893-1905, 2015.

[35] X.-M. Zhang and Q.-L. Han, "Abel lemma-based finite-sum inequality and its application to stability analysis for linear discrete time-delay systems," Automatica, vol. 57, pp. 199-202, 2015.

[36] Y. Shu, X. Liu, and Y. Liu, "Stability and passivity analysis for uncertain discrete-time neural networks with time-varying delay," Neurocomputing, vol. 173, pp. 1706-1714, 2016.

[37] J. Liu and J. Zhang, "Note on stability of discrete-time timevarying delay systems," IET Control Theory \& Applications, vol. 6, no. 2, pp. 335-339, 2012.

[38] T. Wang, M.-X. Xue, C. Zhang, and S.-M. Fei, "Improved stability criteria on discrete-time systems with time-varying and distributed delays," International Journal of Automation and Computing, vol. 10, no. 3, pp. 260-266, 2013.

[39] Z. Feng, J. Lam, and G. H. Yang, "Optimal partitioning method for stability analysis of continuous/discrete delay systems," International Journal of Robust and Nonlinear Control, vol. 25, no. 4, pp. 559-574, 2013.

[40] L. Jin, Y. He, and M. Wu, "Improved delay-dependent stability analysis of discrete-time neural networks with time-varying delay," Journal of The Franklin Institute, vol. 354, no. 4, pp. 19221936, 2017.

[41] C. Hua, S. Wu, and X. Guan, "New robust stability condition for discrete-time recurrent neural networks with time-varying delays and nonlinear perturbations," Neurocomputing, vol. 219, pp. 203-209, 2017.

[42] X. L. Zhu and G. H. Yang, "Jensen inequality approach to stability analysis of discrete-time systems with time-varying delay," in Proceedings of the American Control Conference, pp. 1644-1649, Seattle, WA, USA, 2008.

[43] A. Seuret, F. Gouaisbaut, and E. Fridman, "Stability of systems with fast-varying delay using improved Wirtinger's inequality," in Proceedings of the 52nd IEEE Conference on Decision and Control (CDC '13), pp. 946-951, IEEE, Florence, Italy, December 2013. 


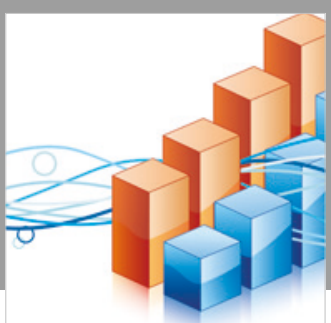

Advances in

Operations Research

\section{-n-m}
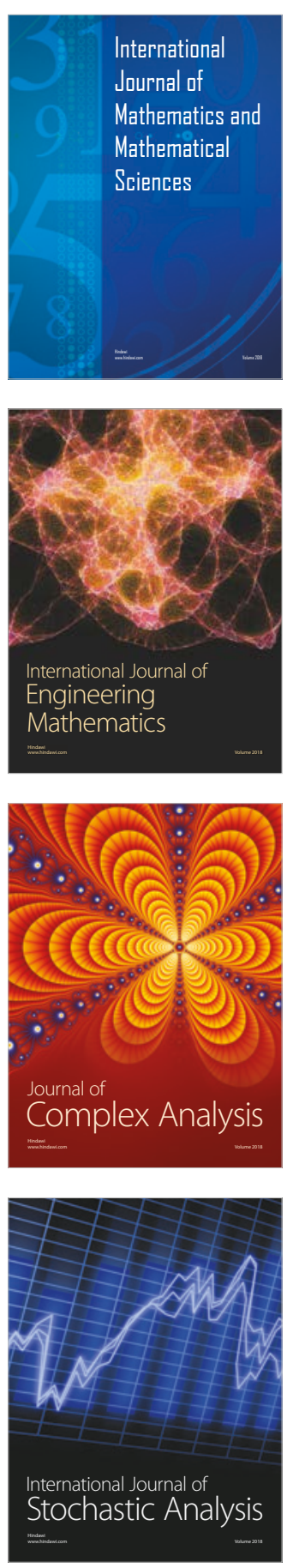
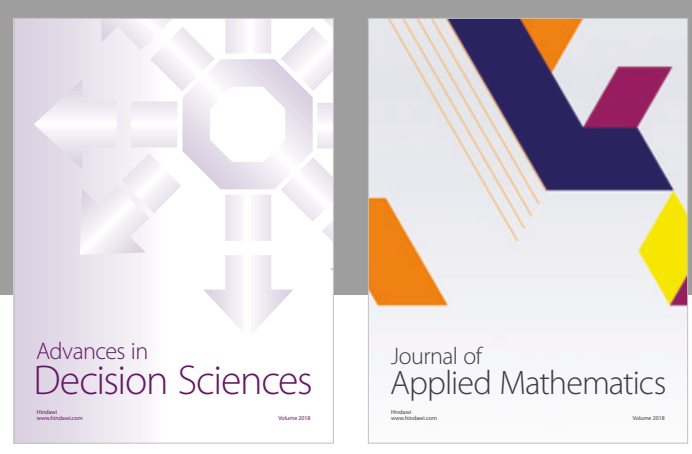

Journal of

Applied Mathematics
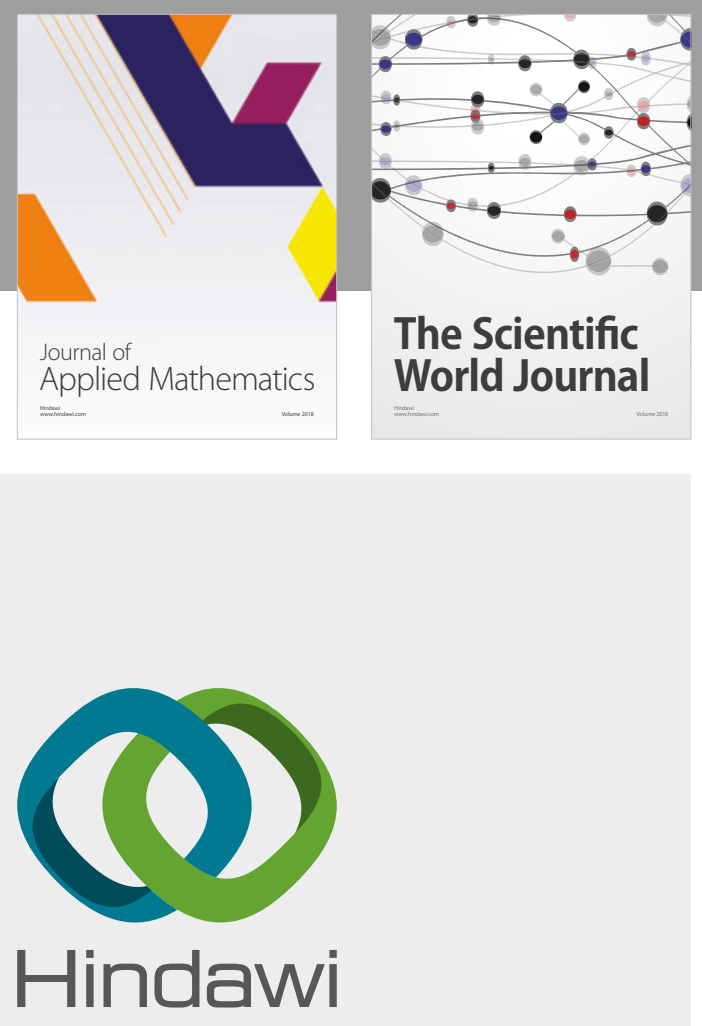

Submit your manuscripts at

www.hindawi.com

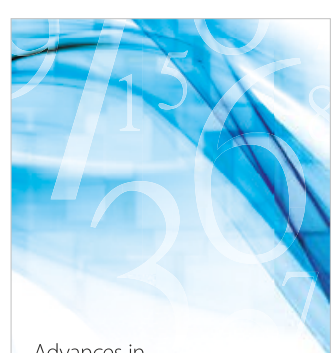

Advances in
Numerical Analysis
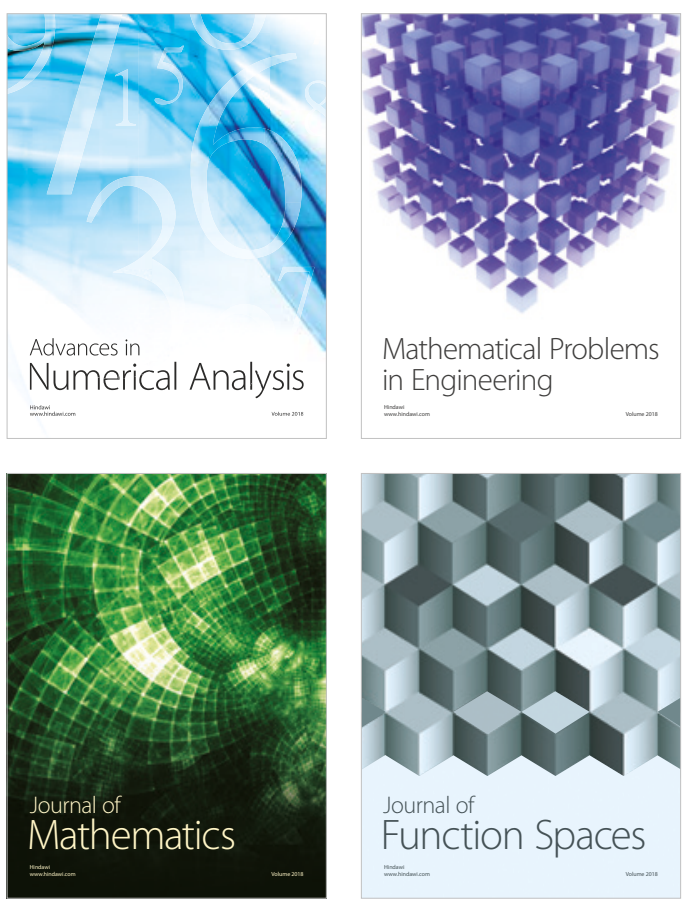

Mathematical Problems in Engineering

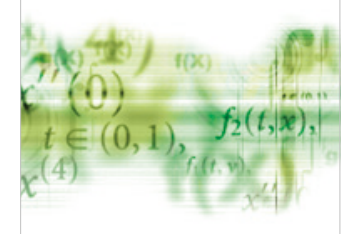

International Journal of

Differential Equations

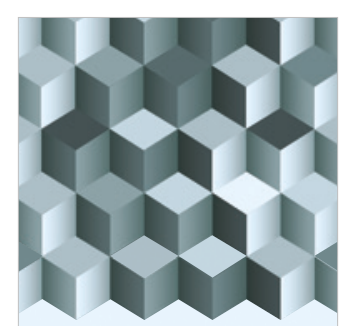

Journal of

Function Spaces

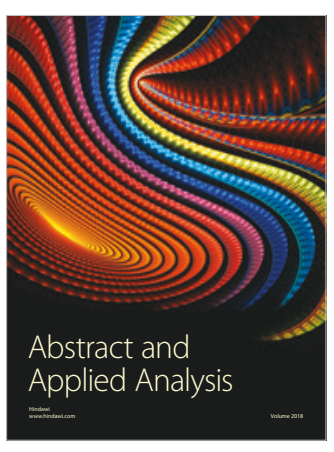

The Scientific

World Journal

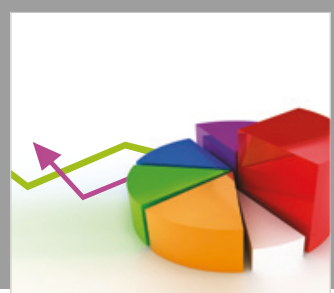

Journal of

Probability and Statistics
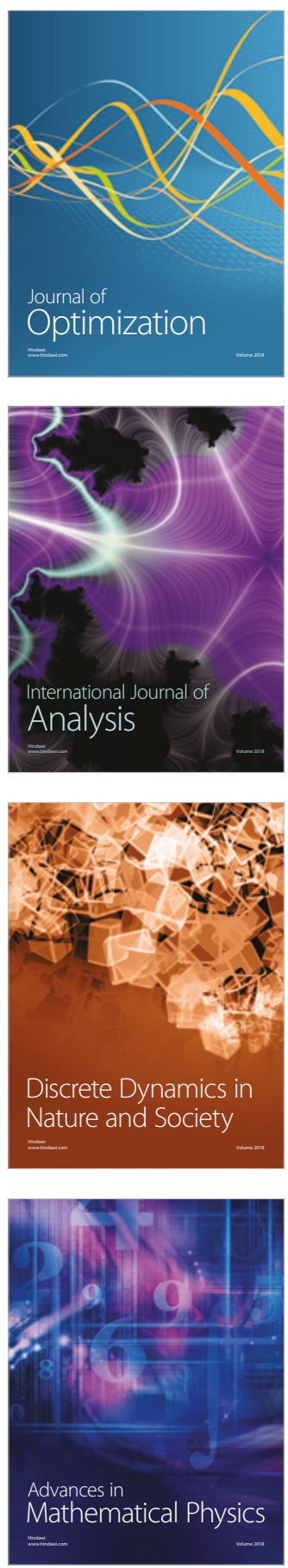